\title{
Search for low mass vector resonances decaying into quark-antiquark pairs in proton-proton collisions at $\sqrt{s}=13 \mathrm{TeV}$
}

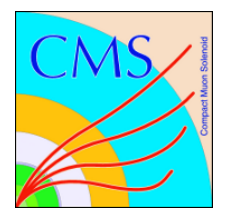

\section{The CMS collaboration}

E-mail: cms-publication-committee-chair@cern.ch

ABSTRACT: A search for narrow vector resonances decaying into quark-antiquark pairs is presented. The analysis is based on data collected in proton-proton collisions at $\sqrt{s}=$ $13 \mathrm{TeV}$ with the CMS detector at the LHC, corresponding to an integrated luminosity of $35.9 \mathrm{fb}^{-1}$. The hypothetical resonance is produced with sufficiently high transverse momentum that its decay products are merged into a single jet with two-prong substructure. A signal would be identified as a peak over a smoothly falling background in the distribution of the invariant mass of the jet, using novel jet substructure techniques. No evidence for such a resonance is observed within the mass range of $50-300 \mathrm{GeV}$. Upper limits at $95 \%$ confidence level are set on the production cross section, and presented in a mass-coupling parameter space. The limits further constrain simplified models of dark matter production involving a mediator interacting between quarks and dark matter particles through a vector or axial-vector current. In the framework of these models, the results are the most sensitive to date, extending for the first time the search region to masses below $100 \mathrm{GeV}$.

KEYWORDS: Jet substructure, Hadron-Hadron scattering (experiments), Dark matter, Jets

ArXIV EPRINT: 1710.00159 


\section{Contents}

1 Introduction 1

2 CMS detector $\quad 2$

3 Event simulation and selection 3

3.1 Simulated samples 3

3.2 Event reconstruction and selection 3

4 Background estimate $\quad 7$

5 Systematic uncertainties $\quad 9$

$\begin{array}{llr}6 & \text { Results } & 10\end{array}$

$\begin{array}{lll}7 & \text { Summary } & 11\end{array}$

$\begin{array}{ll}\text { A Supplementary materials } & 15\end{array}$

$\begin{array}{ll}\text { The CMS collaboration } & 22\end{array}$

\section{Introduction}

Many extensions of the standard model (SM) predict the existence of new resonances that couple to quarks (q) [1-11]. The first searches for such particles were reported by the UA1 [12] and UA2 [13, 14] experiments using $\sqrt{s}=630 \mathrm{GeV}$ collisions at the CERN Sp $\bar{p} S$, and were extended to larger values of resonance masses by the CDF [15-19] and D0 [20] experiments using $\sqrt{s}=1.8$ and $1.96 \mathrm{TeV}$ collisions at the Fermilab Tevatron. At the CERN LHC, the searches in proton-proton (pp) collisions at $\sqrt{s}=7,8$ and $13 \mathrm{TeV}$ performed by the ATLAS [21-27] and CMS [28-35] Collaborations have mostly focused on the production of heavy particles. For resonance masses below $1 \mathrm{TeV}$, the sensitivity is limited by high trigger thresholds and by the large expected backgrounds, notably from $\mathrm{SM}$ events consisting of jets produced through the strong interaction, referred to here as QCD multijet events.

These difficulties can be avoided by an approach focused on the events where at least one high transverse momentum $\left(p_{\mathrm{T}}\right)$ jet from initial-state radiation (ISR) is produced in association with a light resonance decaying into a q $\bar{q}$ pair. The ISR requirement provides enough energy in the event to satisfy the trigger, either by the ISR jet or by the resonance itself. The minimum $p_{\mathrm{T}}$ of the resonance considered in this search is sufficiently high that the hadronization products of the daughter quarks merge and are reconstructed as a 
single, large-radius jet. The only previous search in this topology to place constraints on resonance masses below $300 \mathrm{GeV}$ was by the CMS Collaboration, applying this technique to data collected at the LHC in 2015 [36].

In the current paper, the results of a search for leptophobic vector resonances $\left(Z^{\prime}\right)$ decaying to quark-antiquark pairs in pp collisions at $\sqrt{s}=13 \mathrm{TeV}$ are reported, using data collected by the CMS detector in 2016, corresponding to an integrated luminosity of $35.9 \mathrm{fb}^{-1}$. The search is performed by looking for a narrow resonance peak in the continuous jet mass distribution. The analysis exploits a new substructure variable that is decorrelated from the jet mass and $p_{\mathrm{T}}$ and preserves the shape of the jet mass distribution used in the search. The jet is required to have the two-prong substructure expected from the signal. The dominant background from SM QCD multijet production is estimated from a signal-depleted control region created by inverting the substructure requirement. The signal yield is extracted by simultaneously fitting the signal and control regions, while requiring that the ratio of $\mathrm{QCD}$ components in each region is described by a smooth twodimensional function of jet mass and $p_{\mathrm{T}}$. The $\mathrm{W}+$ jets and $\mathrm{Z}+$ jets background components are estimated from simulation and the top quark background contribution is obtained from simulation corrected with scale factors derived from a t $\overline{\mathrm{t}}$-enriched control sample.

Results are interpreted within the framework of a leptophobic vector resonance model, and are also used to set limits on the existence of generic vector-like resonances decaying into quarks [37]. Limits are also set in the context of a simplified model of dark matter (DM) production at the LHC, in which the mediators couple only to quarks and DM particles [38].

\section{CMS detector}

The central feature of the CMS apparatus is a superconducting solenoid of $6 \mathrm{~m}$ internal diameter, providing a magnetic field of $3.8 \mathrm{~T}$. Within the solenoid volume are a silicon pixel and strip tracker, a lead tungstate crystal electromagnetic calorimeter (ECAL), and a brass and scintillator hadron calorimeter (HCAL), each composed of a barrel and two endcap sections. Forward calorimeters extend the pseudorapidity $(\eta)$ coverage provided by the barrel and endcap detectors. Muons are detected in gas-ionization chambers embedded in the steel flux-return yoke outside the solenoid.

Events are selected using a two-tiered trigger system [39]. The first level, composed of custom hardware processors, uses information from the calorimeters and muon detectors to select events of interest in a time interval of less than $4 \mu \mathrm{s}$. The second level, known as the high-level trigger (HLT), consists of a farm of processors running a version of the full event reconstruction software optimized for fast processing, and further reduces the event rate from around $100 \mathrm{kHz}$ to less than $1 \mathrm{kHz}$, before data storage.

A more detailed description of the CMS detector, together with a definition of the coordinate system used and the relevant kinematic variables, can be found in ref. [40]. 


\section{Event simulation and selection}

\subsection{Simulated samples}

Simulated samples of the $\mathrm{Z}^{\prime}$ resonance decaying into a quark-antiquark pair are generated at leading order (LO) with the MADGRAPH5_aMC@NLO 2.2.3 generator [41] with up to 3 extra jets in matrix element calculations. The dominant SM backgrounds arise from multijet and $\mathrm{W} / \mathrm{Z}+$ jets processes. These backgrounds are simulated at LO using the MADGRAPH5_aMC@NLO generator with the MLM matching [42] between jets from matrix element calculations and from parton showers, while the POWHEG 2.0 [43] generator at nextto-leading order (NLO) precision is used to model the subdominant contribution from pair and single top quark production. All signal and background generators are interfaced with PYTHIA 8.212 [44], with the CUETP8M1 underlying event tune [45], to simulate parton showering and hadronization effects. The generated events are further processed through a GEANT4 [46] simulation of the CMS detector. The parton distribution function (PDF) set NNPDF3.0 [47] is used to produce all simulated samples, with the accuracy (LO or NLO) determined by the generator used. For events containing $\mathrm{W}$ and $\mathrm{Z}$ bosons, we apply higher-order QCD and electroweak $(\mathrm{EW}) p_{\mathrm{T}}$ dependent corrections to improve the modeling of the $p_{\mathrm{T}}$ distribution of $\mathrm{W}$ and $\mathrm{Z}$ events, following refs. [48-52]. The same NLO $\mathrm{QCD}$ corrections that are applied to the $\mathrm{W}$ and $\mathrm{Z}$ simulation are also applied to the signal simulation. However, since the coupling of the $\mathrm{Z}^{\prime}$ mediator differs from that of the $\mathrm{Z}$ boson, the equivalent Z NLO EW corrections are not applied to the signal model.

\subsection{Event reconstruction and selection}

The CMS particle-flow (PF) event algorithm [53] reconstructs and identifies individual particles with an optimized combination of information from the various elements of the CMS detector. Each particle candidate is classified as either an electron, a muon, a photon, or a charged or neutral hadron. The energy of photons is obtained directly from the ECAL measurement, corrected for zero-suppression effects. The energy of electrons is determined from a combination of the electron momentum at the primary interaction vertex as determined by the tracker, the energy of the corresponding ECAL cluster, and the energy sum of all bremsstrahlung photons spatially compatible with originating from the electron track. The energy of muons is obtained from the curvature of the corresponding track. The energy of charged hadrons is determined from a combination of their momentum measured in the tracker and the matching ECAL and HCAL energy deposits, corrected for zerosuppression effects and for the response function of the calorimeters to hadronic showers. Finally, the energy of neutral hadrons is obtained from the corresponding corrected ECAL and HCAL energy. The missing transverse momentum vector is defined as the negative vectorial sum of the transverse momenta of all the particles identified in the event, and its magnitude is referred to as $p_{\mathrm{T}}^{\text {miss }}$.

The PF candidates are clustered into jets using the anti- $k_{\mathrm{T}}$ algorithm $[54,55]$. Jets are clustered with distance parameters of 0.4 and 0.8 , and are referred to as AK4 and AK8 jets, respectively. To mitigate the impact of particles arising from additional proton-proton interactions within the same or adjacent bunch crossings (pileup), weights calculated with 
the pileup-per-particle identification algorithm [56] are applied to each PF candidate prior to jet clustering, based on the likelihood of it coming from the hard-scattering vertex. Further corrections are applied to simulated jet energies as a function of jet $\eta$ and $p_{\mathrm{T}}$ to match the observed detector response [57, 58].

This search focuses on events in which a high- $p_{\mathrm{T}}$ jet from a merged $Z^{\prime} \rightarrow q \bar{q}$ recoils against another high- $p_{\mathrm{T}}$ ISR jet. A combination of several online signatures is required for the trigger selection, all requiring the total hadronic transverse energy in the event $\left(H_{\mathrm{T}}\right)$ or the jet $p_{\mathrm{T}}$ to exceed a certain threshold. In addition, soft radiation remnants are removed with the jet trimming technique [59] before the mass selection, allowing the $H_{\mathrm{T}}$ and jet $p_{\mathrm{T}}$ trigger thresholds to be reduced, and improving the signal acceptance. To be fully efficient with respect to the trigger requirement, we require at least one AK8 jet with $p_{\mathrm{T}}>500 \mathrm{GeV}$ and $|\eta|<2.5$. Additional quality criteria are applied to the jets in order to remove spurious jet-like features originating from isolated noise patterns in the calorimeters or the tracker. The efficiency of these jet quality requirements for signal events is above 99\%. In order to reduce backgrounds from SM EW processes, events are removed if they contain identified and isolated electrons, muons, or taus with $p_{\mathrm{T}}>10 \mathrm{GeV}$ and $|\eta|<2.5$, 2.4 , or 2.3 , respectively, according to the isolation criteria in [48].

In the subsequent offline analysis, the most energetic jet in the event is assumed to correspond to the $\mathrm{Z}^{\prime} \rightarrow \mathrm{q} \overline{\mathrm{q}}$ system, and is reconstructed as a single AK8 jet. The search is performed using the distribution of the jet mass groomed with the soft-drop algorithm $\left(m_{\mathrm{SD}}\right)$, which is an extension of the modified mass drop tagger $[60,61]$ that removes soft and wide-angle radiation produced by parton shower activity, pileup interactions, and the underlying event from the jet. Jets are groomed using the parameters $z_{\text {cut }}=0.1$ and $\beta=0$. Here, $z_{\text {cut }}$ specifies subleading the energy fraction relative to the whole jet at which jet declustering into subjet pairs is stopped. The parameter $\beta$ adds additional angular requirements on the jet declustering. For $\beta=0$, these requirements are neglected, and approximately the same fraction of energy is groomed away regardless of the initial jet energy [61]. The soft-drop grooming reduces the jet mass for QCD background jets when large masses arise from soft gluon radiation. In contrast, the jet mass for merged $Z^{\prime} \rightarrow \mathrm{q} \bar{q}$ and $\mathrm{W} / \mathrm{Z} \rightarrow \mathrm{q} \overline{\mathrm{q}}$ jets comes from the kinematic distributions of the decay, and is largely unchanged by grooming. Figure 1 shows the distributions of $m_{\mathrm{SD}}$ for data and simulation, after the jet kinematic selection.

In this paper, the dimensionless scaling variable $\rho$ [60, 62], defined as $\rho=\ln \left(m_{\mathrm{SD}}^{2} / p_{\mathrm{T}}^{2}\right)$, is used in the characterization of the correlation of jet substructure variables with the jet mass and $p_{\mathrm{T}}$. For QCD jets, the distribution of $\rho$ is approximately invariant under a change of jet $p_{\mathrm{T}}$, in the region where perturbative contributions dominate and scale as $\left(m_{\mathrm{SD}} / p_{\mathrm{T}}\right)$. This property does not hold in two regimes: in the low mass region below $\rho \approx-6$, where non-perturbative effects are large and scale as $\left(1 / m_{\mathrm{SD}}\right)$ instead, and in the high mass region above $\rho \approx-2$. The departure from $\rho$ invariance in the latter case arises because the cone size of the AK8 jets is insufficient to provide complete containment at high masses. Consequently, only events in the range $-5.5<\rho<-2.0$ are considered. This requirement is fully efficient for the $\mathrm{Z}^{\prime}$ boson signal and roughly translates to a $m_{\mathrm{SD}}$ range from $25 \mathrm{GeV}$ to $185 \mathrm{GeV}$ at $p_{\mathrm{T}}=500 \mathrm{GeV}$. 


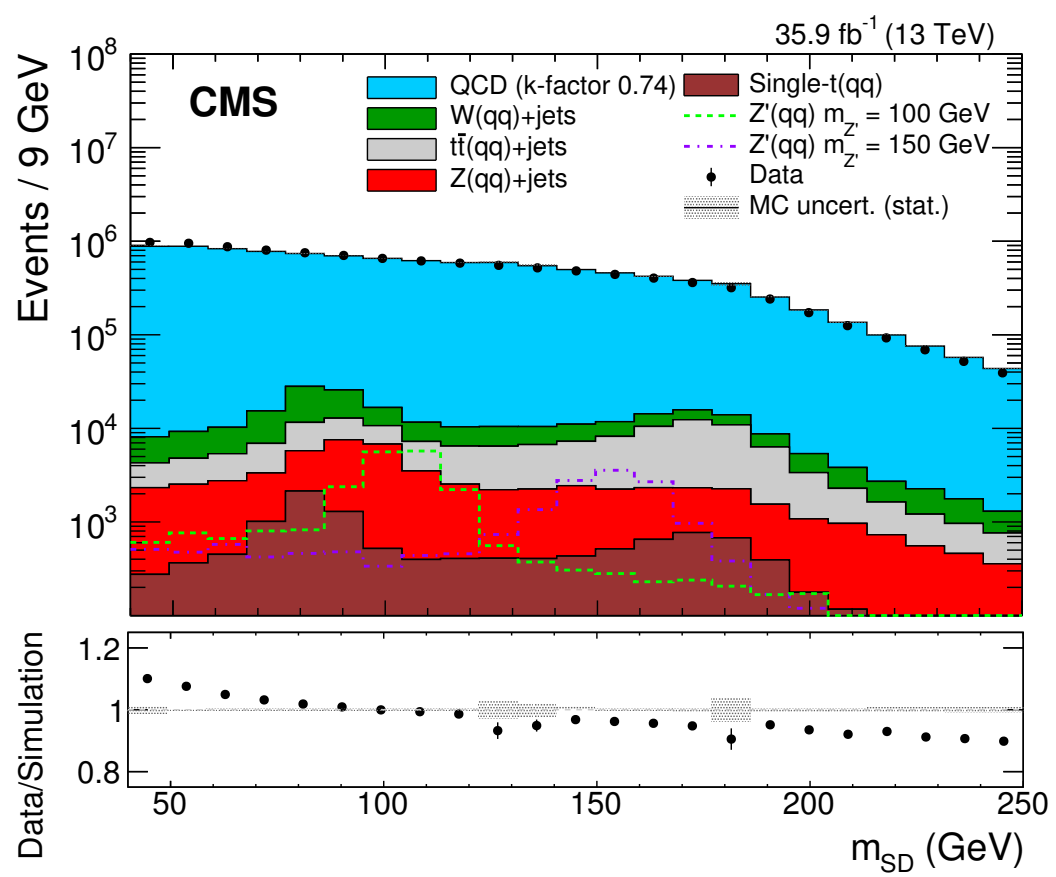

Figure 1. Distributions of data (points) and simulated backgrounds (histograms), of the leading $p_{\mathrm{T}}$ jet soft-drop mass after the jet kinematic selection. Dashed lines illustrate the signal contribution for different $\mathrm{Z}^{\prime}$ boson masses. The multijet processes (QCD) dominate the background component, with subdominant contributions from inclusive SM W, Z, and t $\overline{\mathrm{t}}$ and single top quark processes. The QCD simulation is corrected by an overall factor of 0.74 to match the data yield.

In addition to the jet mass, the observable $N_{2}^{1}$ [63] is used to discriminate the two-prong structure of the jets from the $\mathrm{Z}^{\prime} \rightarrow \mathrm{q} \overline{\mathrm{q}}$ decay from the hadronization products of single light quarks or gluons, which are overwhelmingly one-prong. This jet substructure variable is defined from a combination of generalized energy correlation functions ${ }_{v} e_{n}$, sensitive to correlations of $v$ pairwise angles among $n$-jet constituents [63]. In particular, the 2-point $\left({ }_{1} e_{2}\right)$ and 3-point $\left({ }_{2} e_{3}\right)$ correlation functions are defined as:

$$
\begin{aligned}
{ }_{1} e_{2} & =\sum_{1 \leq i<j \leq n} z_{i} z_{j} \Delta R_{i j}, \\
{ }_{2} e_{3} & =\sum_{1 \leq i<j<k \leq n} z_{i} z_{j} z_{k} \min \left\{\Delta R_{i j} \Delta R_{i k}, \Delta R_{i j} \Delta R_{j k}, \Delta R_{i k} \Delta R_{j k}\right\},
\end{aligned}
$$

where $z_{i}$ represents the energy fraction of the constituent $i$ in the jet and $\Delta R_{i j}$ is the angular separation between constituents $i$ and $j$. For a two-prong structure, signal jets have a stronger 2-point correlation than a 3 -point correlation. The discriminant variable $N_{2}^{1}$ is then constructed via the ratio:

$$
N_{2}^{1}=\frac{{ }_{2} e_{3}}{\left({ }_{1} e_{2}\right)^{2}} .
$$

The energy correlation functions are computed from the jet constituents after the softdrop grooming has been applied, thereby reducing their dependence on the jet mass and $p_{\mathrm{T}}[63]$. 


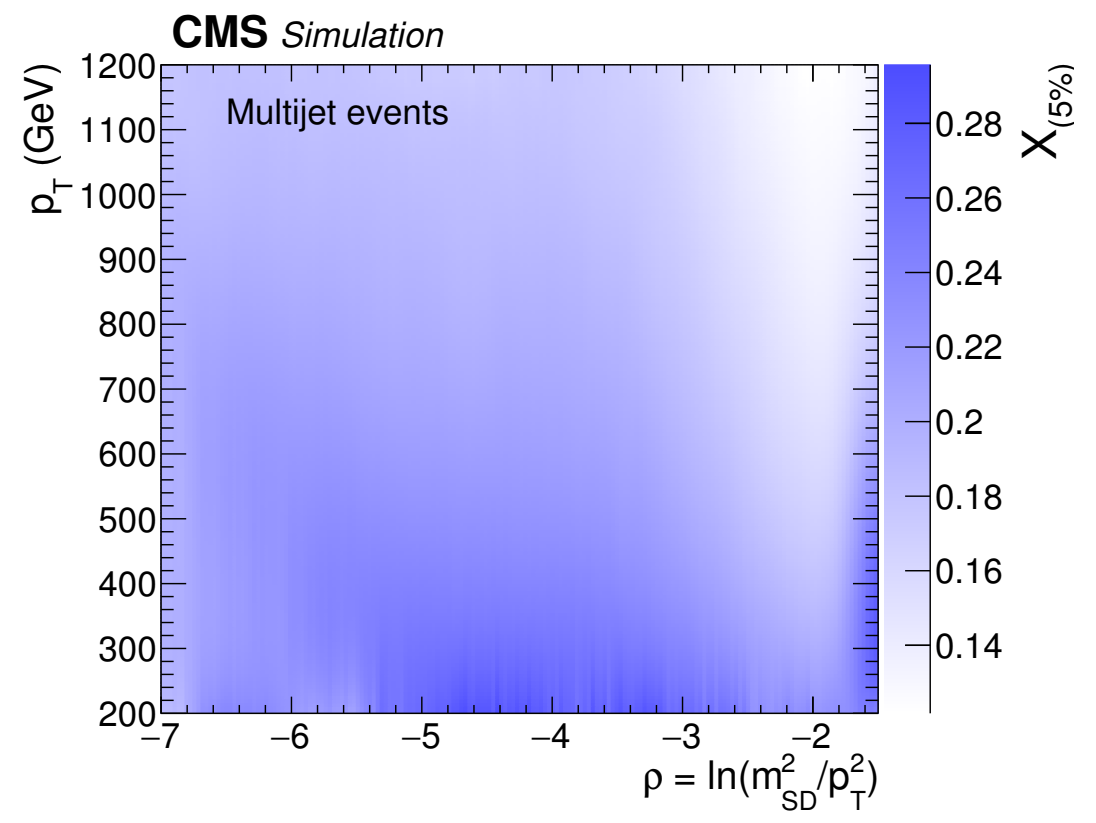

Figure 2. The distribution of $X_{(5 \%)}$ used to define the $N_{2}^{1, \mathrm{DDT}}$ variable, corresponding to the $5 \%$ quantile of the $N_{2}^{1}$ distribution in simulated multijet events. The distribution is shown as a function of the jet $\rho$ and $p_{\mathrm{T}}$ and smoothed using a $\mathrm{kNN}$ approach [64]. The $N_{2}^{1}$ distribution is mostly insensitive to the jet $\rho$ and $p_{\mathrm{T}}$ in the kinematic phase space considered for this analysis $(-5.5<\rho<-2.0)$. Residual correlations in simulation are corrected by applying a decorrelation procedure that yields the $N_{2}^{1, \mathrm{DDT}}$ variable.

The $N_{2}^{1}$ observable has excellent performance in discriminating two-prong signal jets from multijet QCD background jets [63]. However, $N_{2}^{1}$ and similar variables are correlated with the jet mass and $p_{\mathrm{T}}$. A selection based on $N_{2}^{1}$ would distort the jet mass distribution, with the amount of distortion depending on the $p_{\mathrm{T}}$ of the jet. This would make the search for a resonant peak in the jet mass distribution, over a large range of $p_{\mathrm{T}}$, particularly challenging.

The key feature of our approach is that the application of the substructure requirement preserves the shape of the soft-drop jet mass distribution. Improving on the decorrelation procedure proposed in ref. [62], we apply a DDT (designed decorrelated tagger) transformation of $N_{2}^{1}$ to $N_{2}^{1, \mathrm{DDT}}$. It is defined as $N_{2}^{1, \mathrm{DDT}}\left(\rho, p_{\mathrm{T}}\right) \equiv N_{2}^{1}\left(\rho, p_{\mathrm{T}}\right)-X_{(5 \%)}\left(\rho, p_{\mathrm{T}}\right)$, where $X_{(5 \%)}$ is derived from the simulated $N_{2}^{1}$ distribution and illustrated in figure 2 . We require events to pass the $N_{2}^{1, \mathrm{DDT}}\left(\rho, p_{\mathrm{T}}\right)<0$ selection, such that we select a fixed $5 \%$ of QCD multijet events independent of $\rho$ and $p_{\mathrm{T}}$. The distribution of $X_{(5 \%)}$ is smoothed using a distance weighted k-nearest neighbor $(\mathrm{kNN})$ approach [64]. The chosen percentile maximizes the sensitivity to the $\mathrm{Z}^{\prime}$ boson signal.

The distributions of $N_{2}^{1, \text { DDT }}$ for data and simulation are shown in figure 3 after the jet $p_{\mathrm{T}}>500 \mathrm{GeV}$ requirement. Since there is a visible disagreement between simulation and data, the multijet background is estimated from data, as described in the next section. Additional distributions of kinematic observables for data and simulation are available in appendix A. 


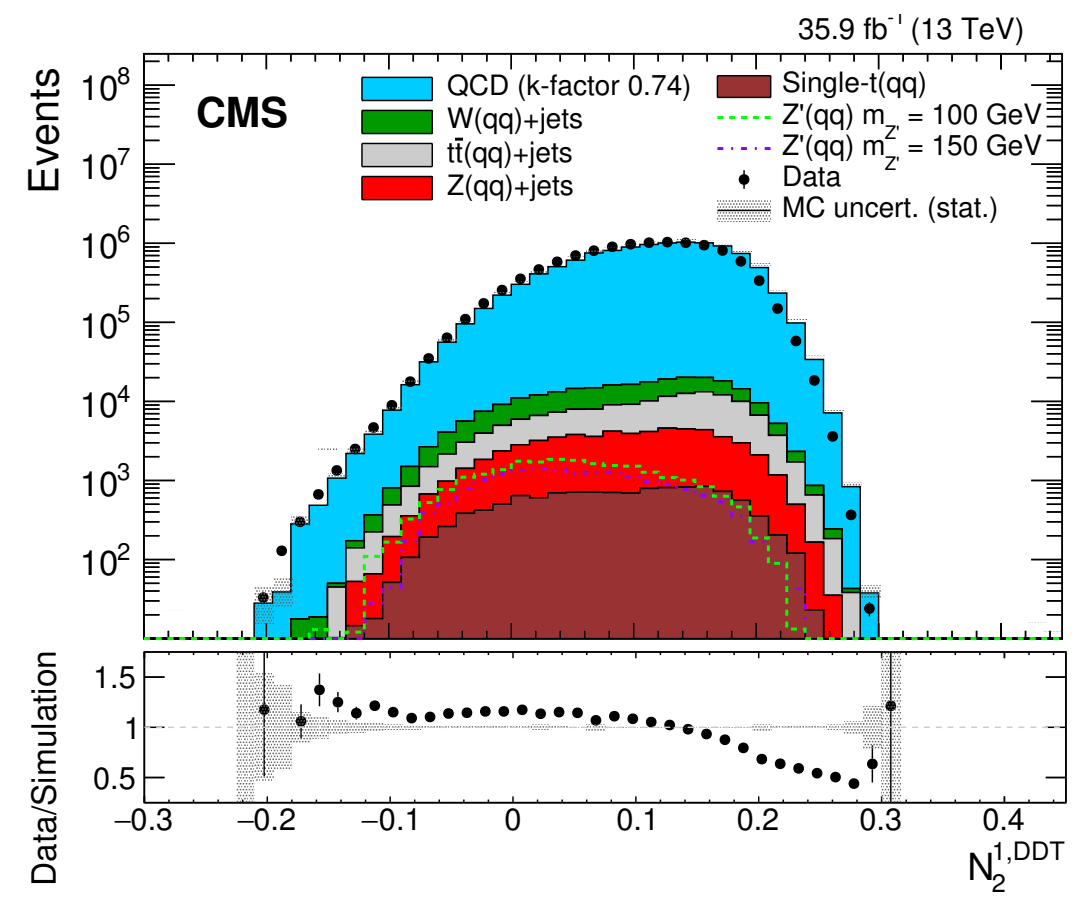

Figure 3. Distributions of data (points) and simulated backgrounds (histograms), of the $N_{2}^{1, \text { DDT }}$ variable for the leading $p_{\mathrm{T}}$ jet after the kinematic selection. Dashed lines illustrate the signal contribution for different $\mathrm{Z}^{\prime}$ boson masses. The multijet processes (QCD) dominate the background component, with subdominant contributions from inclusive SM W, Z, and t $\overline{\mathrm{t}}$ and single top quark processes. The QCD simulation is corrected by an overall factor of 0.74 to match the data yield.

\section{Background estimate}

The search is performed by looking for a resonance in the soft-drop mass distribution over background contributions dominated by QCD multijet events and smaller contributions from $\mathrm{W}\left(\mathrm{q}^{\prime} \overline{\mathrm{q}}\right)+$ jets, $\mathrm{Z}(\mathrm{q} \overline{\mathrm{q}})+$ jets, and top quark background processes.

To model the background contribution from pair and single top quark production we utilize simulation with data-driven corrections based on a dedicated control region. This region has the same kinematic requirements as the signal region but with the muon veto inverted. The muon is selected using dedicated muon triggers and is required to have $p_{\mathrm{T}}>100 \mathrm{GeV}$ and $|\eta|<2.1$ and to be in the opposite hemisphere to the selected AK8 jet. To enrich the $t \bar{t}$ contribution and reduce the multijet contamination, at least one AK4 jet with $p_{\mathrm{T}}>50 \mathrm{GeV}$ is required to pass the b-tagging medium selection based on the combined secondary vertices version-2 algorithm [65], which identifies AK4 jets that originate from the hadronization of b quarks. Separate scale factors correct the overall top quark background normalization and the $N_{2}^{1, \mathrm{DDT}}$ efficiency for mistagging jets from top quark decays. These scale factors are $\mathrm{SF}_{\text {norm }}^{\mathrm{tt}}=0.75 \pm 0.10$ and $\mathrm{SF}_{\text {mistag }}^{\mathrm{t}}=0.83 \pm 0.03$, respectively.

Subdominant backgrounds arising from resonant SM processes $(\mathrm{W} / \mathrm{Z}+$ jets) are estimated from simulations that include corrections to the shape and normalization from higher order NLO QCD and EW calculations. Additional data-to-simulation corrections 

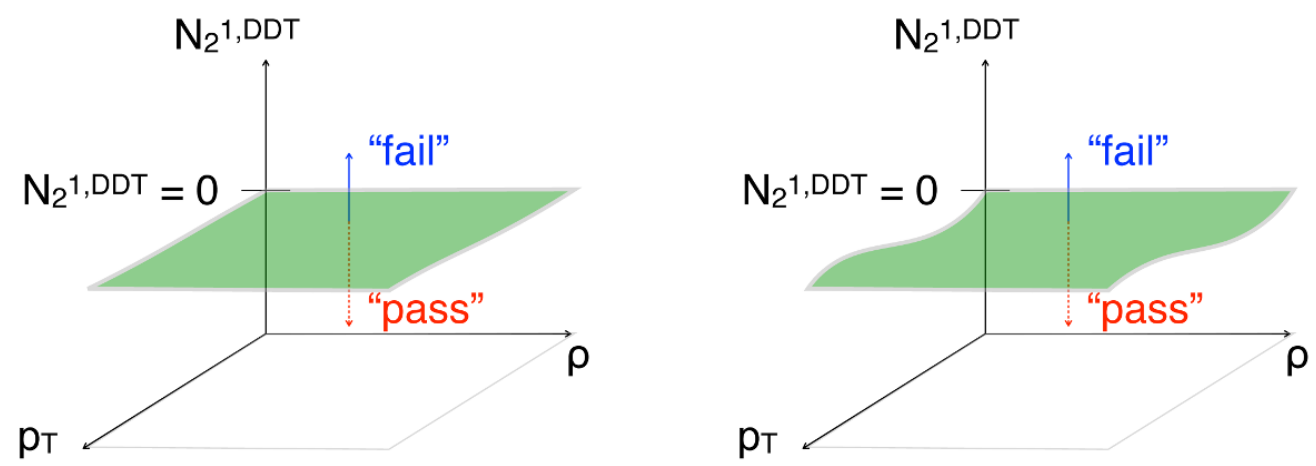

Figure 4. A schematic of the background estimation method. The pass-to-fail ratio, $R_{\mathrm{p} / \mathrm{f}}\left(\rho\left(m_{\mathrm{SD}}, p_{\mathrm{T}}\right)\right)$, is defined from the events passing and failing the $N_{2}^{1, \mathrm{DDT}}$ selection. The variable $N_{2}^{1, \text { DDT }}$ is constructed so that, for simulated multijet events, $R_{\mathrm{p} / \mathrm{f}}$ is constant (left). To account for residual differences between data and simulation, $R_{\mathrm{p} / \mathrm{f}}$ is extracted by performing a two-dimensional fit to data in $\left(\rho, p_{\mathrm{T}}\right)$ space (right).

for the jet mass shapes and $N_{2}^{1, \mathrm{DDT}}$ tagging efficiencies are applied to the simulation. These corrections are evaluated from a t $\overline{\mathrm{t}}$ control region rich in merged hadronic $\mathrm{W}$ bosons, as further explained below.

We estimate the main QCD multijet event background by taking advantage of the decorrelation of $N_{2}^{1, \mathrm{DDT}}$ from $\rho$ and $p_{\mathrm{T}}$. The fraction of events passing the $N_{2}^{1, \mathrm{DDT}}$ selection is, by construction, a constant $5 \%$ in simulated multijet events. The decorrelation ensures that the events passing and failing the selection have the same shape of the QCD jet mass distribution, and their ratio, the "pass-to-fail ratio" $R_{\mathrm{p} / \mathrm{f}}$, is constant for simulated multijet events. The prediction of events passing the selection can then be expressed as:

$$
n_{\mathrm{pass}}^{\mathrm{QCD}}\left(m_{\mathrm{SD}}, p_{\mathrm{T}}\right)=R_{\mathrm{p} / \mathrm{f}}\left(\rho\left(m_{\mathrm{SD}}, p_{\mathrm{T}}\right), p_{\mathrm{T}}\right) n_{\text {fail }}^{\mathrm{QCD}}\left(m_{\mathrm{SD}}, p_{\mathrm{T}}\right)
$$

where $n_{\text {pass }}^{\mathrm{QCD}}$ and $n_{\text {fail }}^{\mathrm{QCD}}$ are the number of passing and failing events in a given $m_{\mathrm{SD}}, p_{\mathrm{T}}$ bin. This procedure is illustrated schematically in figure 4. Since the distribution of $\rho$ is expected to be invariant under a change of $p_{\mathrm{T}}, R_{\mathrm{p} / \mathrm{f}}$ is parametrized as a function of $\rho$, which is in turn expressed as a function of $m_{\mathrm{SD}}$ and $p_{\mathrm{T}}$.

Owing to residual differences between data and simulation, the correction $R_{\mathrm{p} / \mathrm{f}}\left(\rho, p_{\mathrm{T}}\right)$ is allowed to deviate from a constant. The deviation is modeled by expanding $R_{\mathrm{p} / \mathrm{f}}\left(\rho, p_{\mathrm{T}}\right)$ into a polynomial series in orders of $\rho$ and $p_{\mathrm{T}}$ :

$$
\begin{aligned}
R_{\mathrm{p} / \mathrm{f}}\left(\rho, p_{\mathrm{T}}\right)= & \epsilon_{\mathrm{QCD}}\left(1+a_{01} p_{\mathrm{T}}+a_{02} p_{\mathrm{T}}^{2}+\cdots\right. \\
& +\left(a_{10}+a_{11} p_{\mathrm{T}}+a_{12} p_{\mathrm{T}}^{2}+\cdots\right) \rho \\
& \left.+\left(a_{20}+a_{21} p_{\mathrm{T}}+a_{22} p_{\mathrm{T}}^{2}+\cdots\right) \rho^{2}+\cdots\right) .
\end{aligned}
$$

The coefficients $\epsilon_{\mathrm{QCD}}$ and $a_{k \ell}$ have no external constraints but are determined from a simultaneous fit to the data events passing and failing the substructure requirement, together with the signal yield. The number of required coefficients in the fit is determined with a Fisher $F$-test on data [66] by iteratively adding polynomial orders. The optimum 
choice is found to be of fourth order in $\rho$ and third order in $p_{\mathrm{T}}$. The fact that $R_{\mathrm{p} / \mathrm{f}}$ varies slowly across the $m_{\mathrm{SD}}-p_{\mathrm{T}}$ domain is essential, since it allows one to estimate the background under a narrow signal resonance based on the events across the whole jet mass range.

\section{$5 \quad$ Systematic uncertainties}

Uncertainties in the multijet background arise from the fit parameter uncertainties in the pass-to-fail ratio fit described in eq. (4.2). The uncertainties in the top quark background normalization $(10 \%)$ and $N_{2}^{1, \text { DDT }}$ mistag $(2 \%)$ scale factors are propagated to the signal extraction through the fit.

The systematic effects for the shapes and normalization of the $\mathrm{W}, \mathrm{Z}$ backgrounds, and signal components are strongly correlated since they are affected by similar systematic mismeasurements. We constrain the jet mass scale, the jet mass resolution, and the $N_{2}^{1, \text { DDT }}$ selection efficiency using a sample of merged $\mathrm{W}$ boson jets in semileptonic t $\overline{\mathrm{t}}$ events in data. In this region, events are required to have an energetic muon with $p_{\mathrm{T}}>100 \mathrm{GeV}$, $p_{\mathrm{T}}^{\text {miss }}>80 \mathrm{GeV}$, a high- $p_{\mathrm{T}}$ AK8 jet with $p_{\mathrm{T}}>200 \mathrm{GeV}$, and a b-tagged AK4 jet separated from the AK8 jet by $\Delta R>0.8$. Using the same $N_{2}^{1, \mathrm{DDT}}$ requirement described above, we define samples with events that pass and fail the selection for merged $\mathrm{W}$ boson jets in data and simulation, shown in figure 5. A simultaneous fit to the two samples is performed in order to extract the selection efficiency of a merged $\mathrm{W}$ jet in simulation and in data. We measure the data-to-simulation scale factor for the $N_{2}^{1, \text { DDT }}$ selection to be $0.88 \pm 0.10$. The mass scale between data and simulation is found to be $1.10 \pm 0.05$. The jet mass resolution data-to-simulation scale factor is measured to be $1.14 \pm 0.06$. These scale factors determine the initial distributions of the jet mass for the $\mathrm{W}, \mathrm{Z}$ boson, and signal and they are further constrained in the fit to data because of the presence of the $\mathrm{W}$ and $\mathrm{Z}$ resonances in the jet mass distribution. To account for potential deviations due to missing higher-order corrections to the simulated boson $p_{\mathrm{T}}$ distributions, uncertainties are assumed in the $\mathrm{W}$ and $\mathrm{Z}$ boson yields that are $p_{\mathrm{T}}$-dependent. An additional systematic uncertainty is included to account for potential differences between the $\mathrm{W}$ and $\mathrm{Z}$ boson higher-order corrections. Finally, uncertainties associated to the jet energy resolution [57], trigger efficiency, lepton veto efficiency, and the integrated luminosity determination [67] are also applied to the $\mathrm{W}, \mathrm{Z}$ boson, and $\mathrm{Z}^{\prime}$ boson signal yields. A quantitative summary of the systematic effects considered is listed in table 1 .

To validate the robustness of the fit, we perform a goodness-of-fit test and bias tests using pseudo-experiments and injecting a simulated signal, for different values of $Z^{\prime}$ boson mass. No significant bias is observed. As a further test of fit robustness, we split the region failing the $N_{2}^{1, D D T}$ selection into two smaller regions mimicking the passing and failing regions in the signal extraction fit. The mimicked passing-like region corresponds to a background efficiency of $60-65 \%$ and the mimicked failing-like region corresponds to an efficiency of $65-100 \%$. We repeat our background estimation procedure on this selection as if the $60-65 \%$ efficiency region were the passing region. We find negligible biases in the fitted signal strength. 

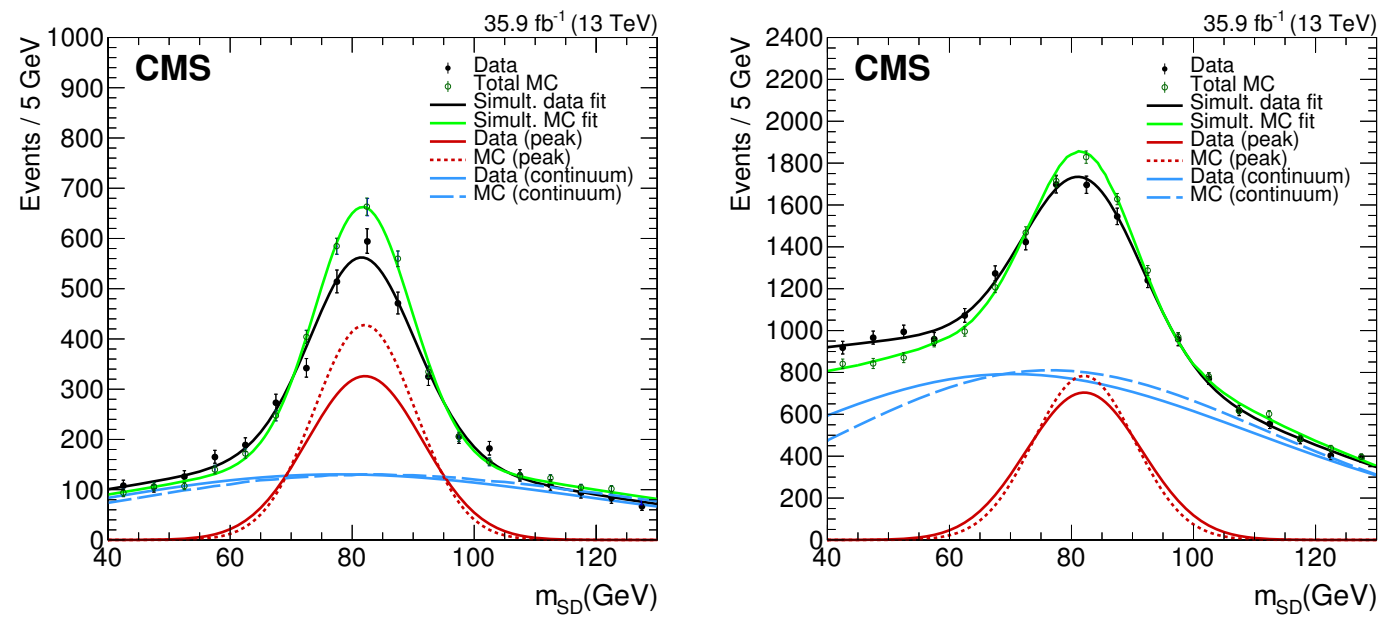

Figure 5. Soft-drop jet mass distributions that pass (left) and fail (right) the $N_{2}^{1, \text { DDT }}$ selection in the semileptonic t $\bar{t}$ sample. Results of fits to data and simulation are shown.

\begin{tabular}{|c|c|c|c|c|}
\hline Systematic source & Multijet & $\mathrm{Z}^{\prime}$ & $\mathrm{W} / \mathrm{Z}$ & $\mathrm{t} \overline{\mathrm{t}}$ \\
\hline Lepton veto efficiency & - & $0.5 \%$ & $0.5 \%$ & - \\
\hline Jet mass scale ${ }^{\dagger}$ & - & $0.5 \%$ & $0.5 \%$ & - \\
\hline Jet mass scale $\left(p_{\mathrm{T}} \text { dependence }\right)^{\dagger} \triangle$ & - & $0.5-2 \%$ & $0.5-2 \%$ & - \\
\hline Trigger efficiency & - & $2 \%$ & $2 \%$ & - \\
\hline Top quark mistag rate & - & - & - & $2 \%$ \\
\hline Integrated luminosity & - & $2.5 \%$ & $2.5 \%$ & - \\
\hline Multijet fit parameters & $1-3 \%$ & - & - & - \\
\hline$N_{2}^{1, \mathrm{DDT}}$ selection efficiency & - & $9 \%$ & $9 \%$ & - \\
\hline Top quark background normalization & - & - & - & $10 \%$ \\
\hline Jet energy resolution $^{\dagger}$ & - & $10 \%$ & $10 \%$ & - \\
\hline NLO QCD corrections & - & $10 \%$ & $10 \%$ & - \\
\hline NLO EW corrections ${ }^{\triangle}$ & - & - & $15-35 \%$ & - \\
\hline NLO EW W/Z decorrelation & - & - & $5-15 \%$ & - \\
\hline
\end{tabular}

Table 1. Summary of the systematic uncertainties for signal and background processes and their relative size. The symbol ${ }^{\triangle}$ denotes uncertainties decorrelated per $p_{\mathrm{T}}$ bin in the $500-1000 \mathrm{GeV}$ range. The symbol ${ }^{\dagger}$ denotes a shape uncertainty in the peaking SM W and Z boson backgrounds and $\mathrm{Z}^{\prime}$ boson signal shape. A long dash (-) indicates that the uncertainty does not apply.

\section{Results}

We combine the estimates of the various SM background processes and search for a potential signal from a $Z^{\prime}$ resonance in the mass range from 50 to $300 \mathrm{GeV}$. A binned maximum likelihood fit to the observed shape of the soft-drop jet mass distribution is performed simultaneously in the passing and failing regions of five $p_{\mathrm{T}}$ ranges whose boundaries are: $500,600,700,800,900$ and $1000 \mathrm{GeV}$. The number of observed events is consistent with the predicted background from SM processes. Figure 6 shows the soft-drop jet mass distribution for data and measured background contributions in the different $p_{\mathrm{T}}$ ranges for a 
$\mathrm{Z}^{\prime}$ mass of $135 \mathrm{GeV}$; the $\mathrm{W}$ and $\mathrm{Z}$ boson contributions are clearly visible in the data. The $m_{\mathrm{SD}}$ distribution for data in the combined $p_{\mathrm{T}}$ ranges is available in appendix A.

The results are interpreted in terms of $95 \%$ confidence level (CL) upper limits on the production cross section. Upper limits are computed using the modified frequentist approach for confidence levels $\left(\mathrm{CL}_{s}\right)$; taking the profile likelihood as the test statistic $[68,69]$ in the asymptotic approximation [70]. They are shown as a function of the resonance mass in figure 7 (left), where they are compared to cross sections for a model of a leptophobic $\mathrm{Z}^{\prime}$ resonance with quark coupling $g_{\mathrm{q}^{\prime}}$ value of either 0.17 or 0.08 that are close to our current sensitivity. Systematic uncertainties are treated as nuisance parameters, which are modeled with log-normal priors and profiled over in the limit calculations. The maximum local observed p-value corresponds to 2.9 standard deviations from the background-only expectation at a $\mathrm{Z}^{\prime}$ boson mass of $115 \mathrm{GeV}$, and the global significance, calculated over the probed mass range [71], corresponds to approximately 2.2 standard deviations.

Upper limits on the signal cross section are translated into the coupling $g_{\mathrm{q}^{\prime}}$ as a function of $\mathrm{Z}^{\prime}$ boson mass, related to the $\mathrm{Z}^{\prime}$ coupling convention of ref. [37] by $g_{\mathrm{q}^{\prime}}=g_{\mathrm{B}} / 6$. Coupling values above the solid curves are excluded. In figure 7 (right), we show previous results from UA2, CDF, ATLAS and CMS experiments. Indirect constraints from the hadronic $\mathrm{Z}$ boson partial width measurement and limits from the UA2 and CDF experiments are interpreted from [37].

The results of this analysis can be used to constrain simplified models of DM. Figure 8 shows the excluded values at $95 \%$ CL of mediator mass $\left(m_{\mathrm{Med}}\right)$ as a function of the dark matter particle mass $\left(m_{\mathrm{DM}}\right)$ for vector mediators, in simplified models that assume a leptophobic mediator that couples only to quarks and DM particles [38, 73]. Limits are shown for a choice of universal quark coupling $g_{\mathrm{q}}=0.25$ and a DM coupling $g_{\mathrm{DM}}=1.0$. The difference in limits between axial-vector and vector mediator couplings is small and thus only constraints for the latter coupling scenario are shown. The excluded range of mediator mass (red) is between 50 and $300 \mathrm{GeV}$. The upper bound decreases to $240 \mathrm{GeV}$ when $m_{\text {Med }}>2 m_{\mathrm{DM}}$, because the branching fraction (BR) to q $\bar{q}$ decreases as the BR to DM becomes kinematically favorable. If $m_{\mathrm{Med}}<2 m_{\mathrm{DM}}$, the mediator cannot decay to DM particles and the dijet cross section from the mediator model becomes identical to that in the leptophobic $\mathrm{Z}^{\prime}$ model, meaning that the limits on the mediator mass in figure 8 are identical to the limits on the $\mathrm{Z}^{\prime}$ mass with a coupling $g_{\mathrm{q}^{\prime}}=g_{\mathrm{q}}=0.25$. For axialvector mediators, the excluded values of mediator mass are expected to be identical to the excluded values in figure 8 when $m_{\mathrm{DM}}>m_{\mathrm{Med}} / 2$ or $m_{D M}=0$, with differences only expected in the transition region $m_{\mathrm{Med}} \simeq 2 m_{\mathrm{DM}}$. Additional limits (blue) in figure 8 come from traditional dijet searches [35].

\section{Summary}

A search for a vector resonance $\left(\mathrm{Z}^{\prime}\right)$ decaying into a quark-antiquark pair and reconstructed as a single jet has been presented, using a data set comprising proton-proton collisions at $\sqrt{s}=13 \mathrm{TeV}$, corresponding to an integrated luminosity of $35.9 \mathrm{fb}^{-1}$. Novel substructure techniques are employed to identify a jet containing a $\mathrm{Z}^{\prime}$ boson candidate over a smoothly 

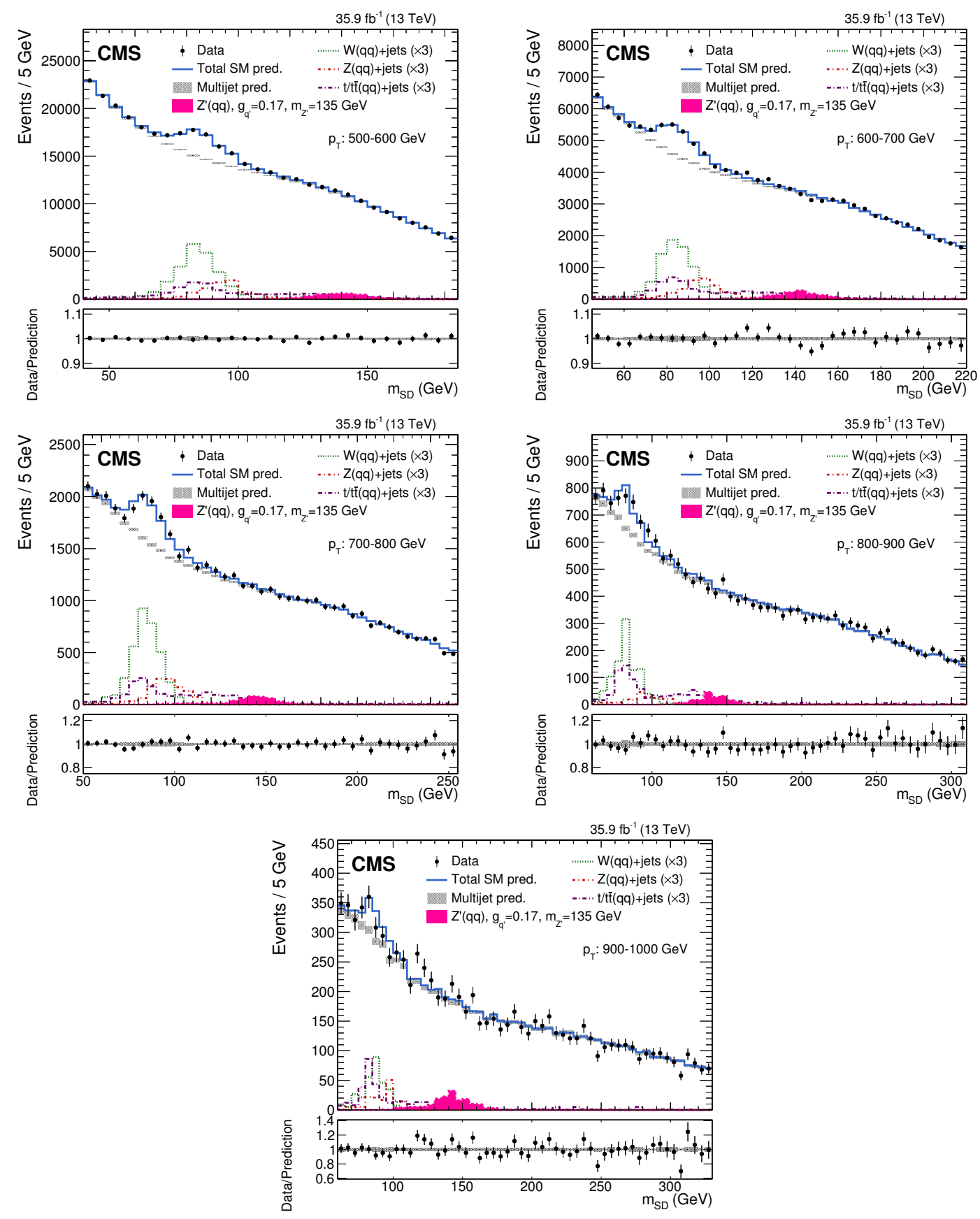

Figure 6. Soft-drop jet mass distribution for the different $p_{\mathrm{T}}$ ranges of the fit from 500 to $1000 \mathrm{GeV}$. Data are shown as black points. The multijet background prediction, including uncertainties, is shown by the shaded bands. Contributions from the $\mathrm{W}$ and $\mathrm{Z}$ boson, and top quark background processes are shown, scaled up by a factor of 3 for clarity. A hypothetical $Z^{\prime}$ boson signal at a mass of $135 \mathrm{GeV}$ is also indicated. In the bottom panel, the ratio of the data to the background prediction, including uncertainties, is shown. The scale on the $\mathrm{x}$-axis differs for each $p_{\mathrm{T}}$ range due to the kinematic selection on $\rho$. 

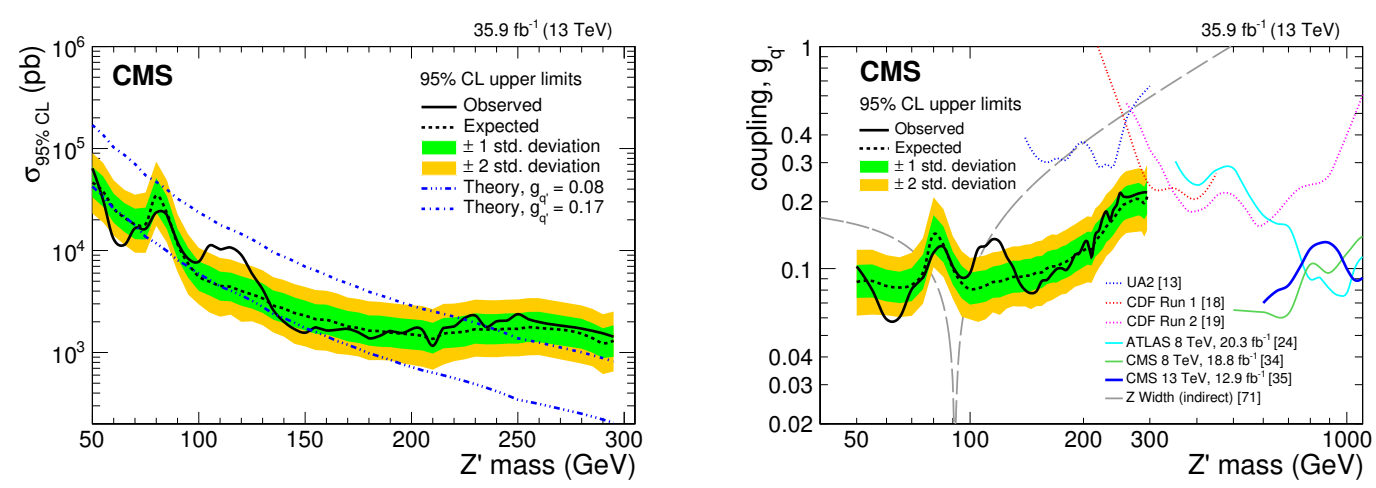

Figure 7. The 95\% CL upper limits on the $\mathrm{Z}^{\prime}$ boson production cross section compared to theoretical cross sections (left) and on the quark coupling $g_{\mathrm{q}^{\prime}}$ as a function of resonance mass for a leptophobic $Z^{\prime}$ resonance that only couples to quarks (right). The observed limits (solid), expected limits (dashed) and their variation at the 1 and 2 standard deviation levels (shaded bands) are shown. Limits from other relevant searches and an indirect constraint on a potential $\mathrm{Z}^{\prime}$ signal from the SM Z boson width [72] are also shown.

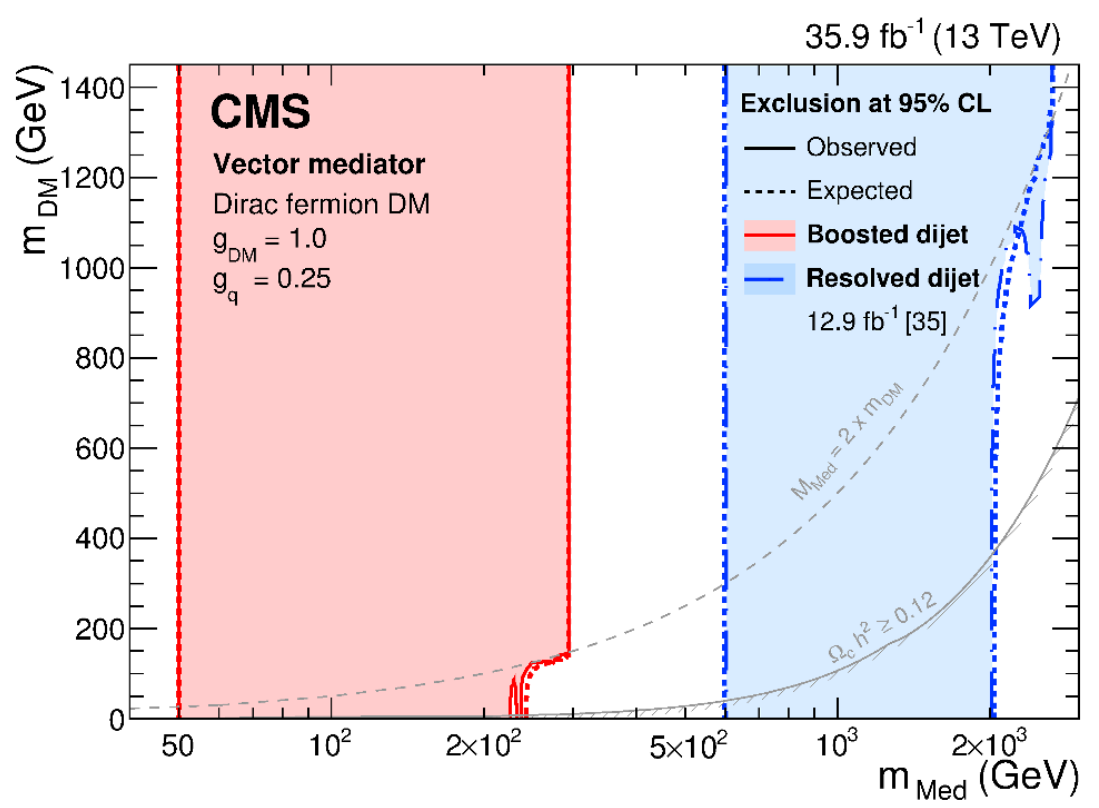

Figure 8. The 95\% CL observed (solid red) and expected (dashed red) excluded regions in the plane of dark matter particle mass $\left(m_{\mathrm{DM}}\right)$ vs. mediator mass $\left(m_{\mathrm{Med}}\right)$, for vector mediators. A branching fraction of $100 \%$ is assumed for a leptophobic vector mediator decaying to dijets. The exclusion is computed for a quark coupling choice $g_{q}=0.25$ and for a dark matter coupling $g_{\mathrm{DM}}=1$. The excluded regions from the dijet resolved analysis (blue dot dashed lines) using early 2016 data [35] are also shown. Results are compared to constraints from the cosmological relic density of DM (light gray) determined from astrophysical measurements [74, 75] and MADDM version 2.0.6 [76, 77] as described in ref. [78]. 
falling soft-drop jet mass distribution in data. No significant excess above the SM prediction is observed, and 95\% confidence level upper limits are set on the $\mathrm{Z}^{\prime}$ boson coupling to quarks, $g_{\mathrm{q}^{\prime}}$, as a function of the $\mathrm{Z}^{\prime}$ boson mass. Coupling values of $g_{\mathrm{q}^{\prime}}>0.25$ are excluded over the $\mathrm{Z}^{\prime}$ mass range from 50 to $300 \mathrm{GeV}$, with strong constraints for masses less than $200 \mathrm{GeV}$. The results obtained for masses from 50 to $100 \mathrm{GeV}$ represent the first direct limits to be published in this range. Limits are set on a simplified model of dark matter mediators that only couple to quarks and dark matter particles, excluding vector mediators with masses between 50 and $300 \mathrm{GeV}$, and using a universal quark coupling $g_{\mathrm{q}}=0.25$ and a dark matter coupling $g_{\mathrm{DM}}=1.0$.

\section{Acknowledgments}

We congratulate our colleagues in the CERN accelerator departments for the excellent performance of the LHC and thank the technical and administrative staffs at CERN and at other CMS institutes for their contributions to the success of the CMS effort. In addition, we gratefully acknowledge the computing centres and personnel of the Worldwide LHC Computing Grid for delivering so effectively the computing infrastructure essential to our analyses. Finally, we acknowledge the enduring support for the construction and operation of the LHC and the CMS detector provided by the following funding agencies: BMWFW and FWF (Austria); FNRS and FWO (Belgium); CNPq, CAPES, FAPERJ, and FAPESP (Brazil); MES (Bulgaria); CERN; CAS, MoST, and NSFC (China); COLCIENCIAS (Colombia); MSES and CSF (Croatia); RPF (Cyprus); SENESCYT (Ecuador); MoER, ERC IUT, and ERDF (Estonia); Academy of Finland, MEC, and HIP (Finland); CEA and CNRS/IN2P3 (France); BMBF, DFG, and HGF (Germany); GSRT (Greece); OTKA and NIH (Hungary); DAE and DST (India); IPM (Iran); SFI (Ireland); INFN (Italy); MSIP and NRF (Republic of Korea); LAS (Lithuania); MOE and UM (Malaysia); BUAP, CINVESTAV, CONACYT, LNS, SEP, and UASLP-FAI (Mexico); MBIE (New Zealand); PAEC (Pakistan); MSHE and NSC (Poland); FCT (Portugal); JINR (Dubna); MON, RosAtom, RAS, RFBR and RAEP (Russia); MESTD (Serbia); SEIDI, CPAN, PCTI and FEDER (Spain); Swiss Funding Agencies (Switzerland); MST (Taipei); ThEPCenter, IPST, STAR, and NSTDA (Thailand); TUBITAK and TAEK (Turkey); NASU and SFFR (Ukraine); STFC (United Kingdom); DOE and NSF (U.S.A.).

Individuals have received support from the Marie-Curie programme and the European Research Council and Horizon 2020 Grant, contract No. 675440 (European Union); the Leventis Foundation; the A. P. Sloan Foundation; the Alexander von Humboldt Foundation; the Belgian Federal Science Policy Office; the Fonds pour la Formation à la Recherche dans l'Industrie et dans l'Agriculture (FRIA-Belgium); the Agentschap voor Innovatie door Wetenschap en Technologie (IWT-Belgium); the Ministry of Education, Youth and Sports (MEYS) of the Czech Republic; the Council of Science and Industrial Research, India; the HOMING PLUS programme of the Foundation for Polish Science, cofinanced from European Union, Regional Development Fund, the Mobility Plus programme of the Ministry of Science and Higher Education, the National Science Center (Poland), contracts Harmonia 2014/14/M/ST2/00428, Opus 2014/13/B/ST2/02543, 2014/15/B/ST2/03998, 
and 2015/19/B/ST2/02861, Sonata-bis 2012/07/E/ST2/01406; the National Priorities Research Program by Qatar National Research Fund; the Programa Severo Ochoa del Principado de Asturias; the Thalis and Aristeia programmes cofinanced by EU-ESF and the Greek NSRF; the Rachadapisek Sompot Fund for Postdoctoral Fellowship, Chulalongkorn University and the Chulalongkorn Academic into Its 2nd Century Project Advancement Project (Thailand); the Welch Foundation, contract C-1845; and the Weston Havens Foundation (U.S.A.).

\section{A Supplementary materials}
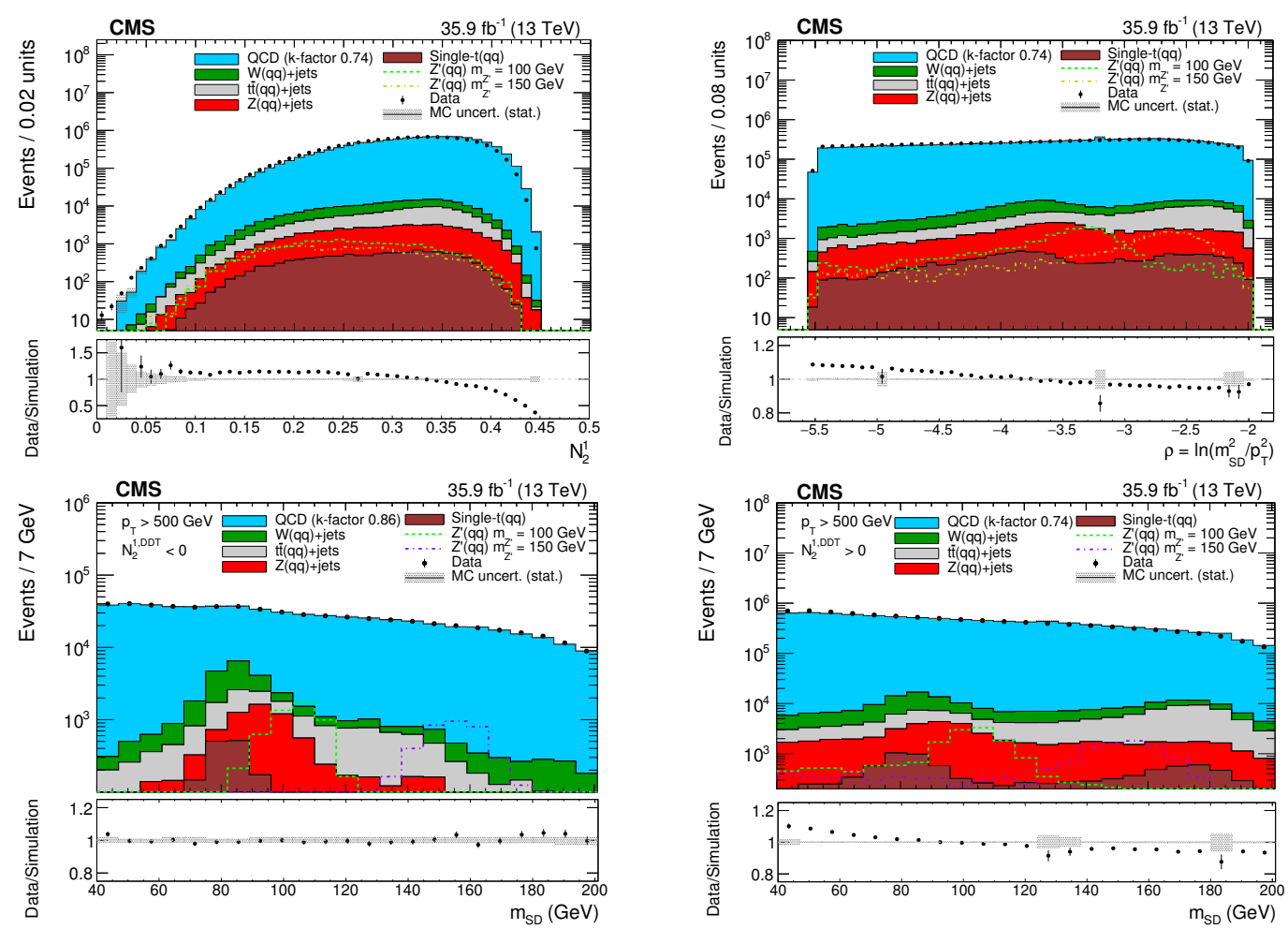

Figure 9. Distributions of data (points) and simulated backgrounds (histograms) of the leading $p_{\mathrm{T}}$ jet $N_{2}^{1}$ (top left) and $\rho$ (top right) observables, after the kinematic selection. The soft-drop jet mass distributions for the passing (bottom left) and failing (bottom right) region, defined by the $N_{2}^{1, \text { DDT }}$ selection, are also shown. The decorrelation ensures that the shape of the multijet mass distribution in both regions is unaffected by the $N_{2}^{1, \mathrm{DDT}}$ selection for different $p_{\mathrm{T}}$ ranges. Dashed lines illustrate the signal contribution for different $Z^{\prime}$ boson masses. The multijet processes (QCD) dominate the background component, with subdominant contributions from inclusive SM W, Z, and $t \bar{t}$ and single top quark processes. The QCD simulation is scaled by an overall factor of 0.74 to match the data yield. Residual differences between data and simulation demonstrate the need for a background estimation method based on control samples in data. 


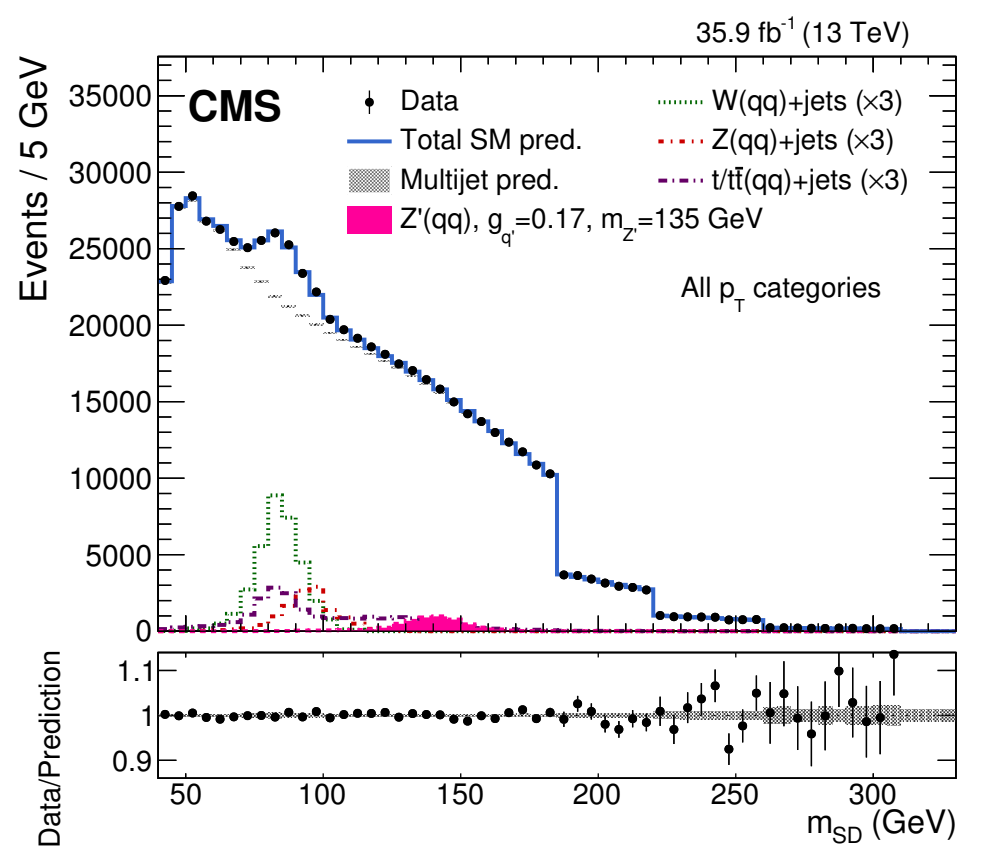

Figure 10. Soft-drop jet mass distribution for the passing region and combined $p_{\mathrm{T}}$ categories. The multijet background prediction in the passing region is obtained using the failing region and the pass-fail ratio $R_{\mathrm{p} / \mathrm{f}}\left(m_{\mathrm{SD}}, p_{\mathrm{T}}\right)$. Data are shown as black points. The multijet background prediction, including uncertainties, is shown by the shaded bands. Contributions from the $\mathrm{W}$ and $\mathrm{Z}$ boson, and top quark background processes are shown, scaled up by a factor of 3 for clarity. A hypothetical $Z^{\prime}$ boson signal at a mass of $135 \mathrm{GeV}$ is also indicated. The features at 45, 185, 220 and $255 \mathrm{GeV}$ in the $m_{\mathrm{SD}}$ distribution are due to the kinematic selection on $\rho$, which affects each $p_{\mathrm{T}}$ category differently. In the bottom panel, the ratio of the data to the background prediction, including uncertainties, is shown.

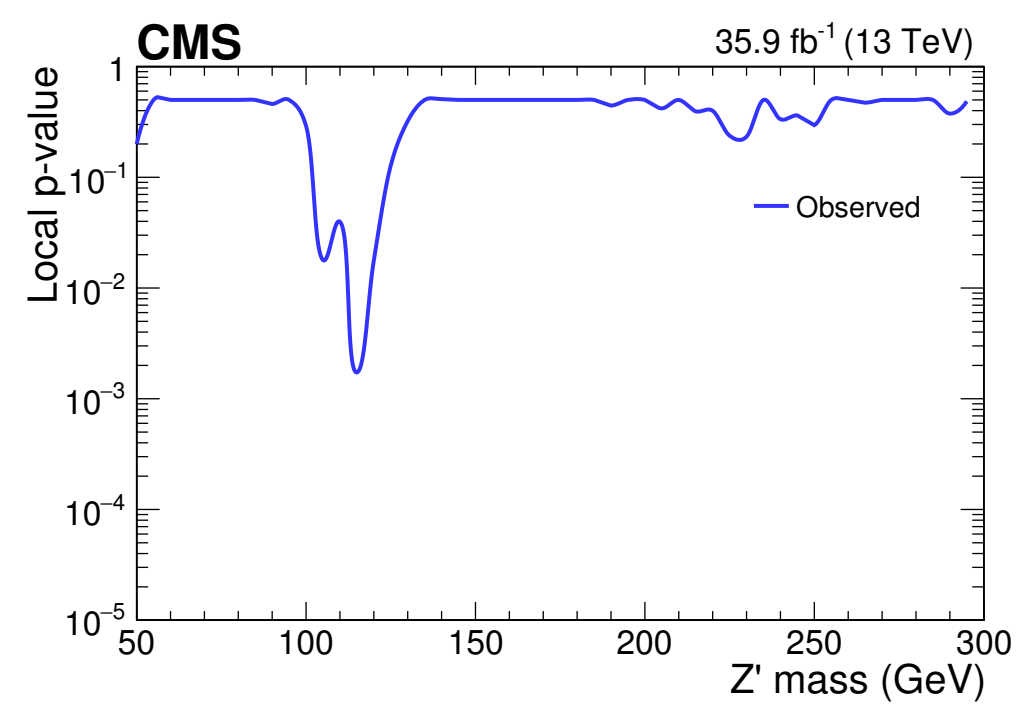

Figure 11. The observed p-value, obtained from the fit to data, as a function of the $\mathrm{Z}^{\prime}$ boson mass. The maximum local observed p-value, at $115 \mathrm{GeV}$, is $1.72 \times 10^{-3}$ and corresponds to 2.9 standard deviations from the background-only expectation, and the global p-value, calculated over the probed mass range, corresponds to 0.0138 and 2.2 standard deviations. 
Open Access. This article is distributed under the terms of the Creative Commons Attribution License (CC-BY 4.0), which permits any use, distribution and reproduction in any medium, provided the original author(s) and source are credited.

\section{References}

[1] L.A. Anchordoqui et al., Dijet signals for low mass strings at the LHC, Phys. Rev. Lett. 101 (2008) 241803 [arXiv:0808.0497] [INSPIRE].

[2] S. Cullen, M. Perelstein and M.E. Peskin, TeV strings and collider probes of large extra dimensions, Phys. Rev. D 62 (2000) 055012 [hep-ph/0001166] [INSPIRE].

[3] J.L. Hewett and T.G. Rizzo, Low-energy phenomenology of superstring inspired $E_{6}$ models, Phys. Rept. 183 (1989) 193 [INSPIRE].

[4] U. Baur, I. Hinchliffe and D. Zeppenfeld, Excited quark production at hadron colliders, Int. J. Mod. Phys. A 2 (1987) 1285 [inSPIRE].

[5] U. Baur, M. Spira and P.M. Zerwas, Excited quark and lepton production at hadron colliders, Phys. Rev. D 42 (1990) 815 [inSPIRE].

[6] P.H. Frampton and S.L. Glashow, Chiral color: an alternative to the standard model, Phys. Lett. B 190 (1987) 157 [inSPIRE].

[7] R.S. Chivukula, A. Farzinnia, E.H. Simmons and R. Foadi, Production of massive color-octet vector bosons at next-to-leading order, Phys. Rev. D 85 (2012) 054005 [arXiv:1111.7261] [INSPIRE].

[8] E.H. Simmons, Coloron phenomenology, Phys. Rev. D 55 (1997) 1678 [hep-ph/9608269] [INSPIRE].

[9] T. Han, I. Lewis and Z. Liu, Colored resonant signals at the LHC: largest rate and simplest topology, JHEP 12 (2010) 085 [arXiv: 1010.4309] [INSPIRE].

[10] E. Eichten, I. Hinchliffe, K.D. Lane and C. Quigg, Super collider physics, Rev. Mod. Phys. 56 (1984) 579 [INSPIRE].

[11] L. Randall and R. Sundrum, An alternative to compactification, Phys. Rev. Lett. 83 (1999) 4690 [hep-th/9906064] [INSPIRE].

[12] UA1 collaboration, C. Albajar et al., Two jet mass distributions at the CERN proton-anti-proton collider, Phys. Lett. B 209 (1988) 127 [INSPIRE].

[13] UA2 collaboration, J. Alitti et al., A measurement of two jet decays of the $W$ and $Z$ bosons at the CERN $\bar{p} p$ collider, Z. Phys. C 49 (1991) 17 [InSPIRE].

[14] UA2 collaboration, J. Alitti et al., A search for new intermediate vector mesons and excited quarks decaying to two jets at the CERN $\bar{p} p$ collider, Nucl. Phys. B 400 (1993) 3 [INSPIRE].

[15] CDF collaboration, F. Abe et al., The two jet invariant mass distribution at $\sqrt{s}=1.8 \mathrm{TeV}$, Phys. Rev. D 41 (1990) 1722 [inSPIRE].

[16] CDF collaboration, F. Abe et al., Search for quark compositeness, axigluons and heavy particles using the dijet invariant mass spectrum observed in pp collisions, Phys. Rev. Lett. 71 (1993) 2542 [INSPIRE].

[17] CDF collaboration, F. Abe et al., Search for new particles decaying to dijets in p p collisions at $\sqrt{s}=1.8 \mathrm{TeV}$, Phys. Rev. Lett. 74 (1995) 3538 [hep-ex/9501001] [INSPIRE].

[18] CDF collaboration, F. Abe et al., Search for new particles decaying to dijets at CDF, Phys. Rev. D 55 (1997) R5263 [hep-ex/9702004] [INSPIRE]. 
[19] CDF collaboration, T. Aaltonen et al., Search for new particles decaying into dijets in proton-antiproton collisions at $\sqrt{s}=1.96$ TeV, Phys. Rev. D 79 (2009) 112002 [arXiv:0812.4036] [INSPIRE].

[20] D0 collaboration, V.M. Abazov et al., Search for new particles in the two jet decay channel with the Do detector, Phys. Rev. D 69 (2004) 111101 [hep-ex/0308033] [INSPIRE].

[21] ATLAS collaboration, Search for new particles in two-jet final states in 7 TeV proton-proton collisions with the ATLAS detector at the LHC, Phys. Rev. Lett. 105 (2010) 161801 [arXiv: 1008.2461] [INSPIRE].

[22] ATLAS collaboration, Search for new physics in dijet mass and angular distributions in $p p$ collisions at $\sqrt{s}=7$ TeV measured with the ATLAS detector, New J. Phys. 13 (2011) 053044 [arXiv: 1103.3864] [INSPIRE].

[23] ATLAS collaboration, Search for new physics in the dijet mass distribution using $1 \mathrm{fb}^{-1}$ of pp collision data at $\sqrt{s}=7 \mathrm{TeV}$ collected by the ATLAS detector, Phys. Lett. B 708 (2012) 37 [arXiv:1108.6311] [INSPIRE].

[24] ATLAS collaboration, ATLAS search for new phenomena in dijet mass and angular distributions using pp collisions at $\sqrt{s}=7$ TeV, JHEP 01 (2013) 029 [arXiv:1210.1718] [INSPIRE].

[25] ATLAS collaboration, Search for new phenomena in the dijet mass distribution using pp collision data at $\sqrt{s}=8$ TeV with the ATLAS detector, Phys. Rev. D 91 (2015) 052007 [arXiv: 1407.1376] [INSPIRE].

[26] ATLAS collaboration, Search for new phenomena in dijet mass and angular distributions from pp collisions at $\sqrt{s}=13$ TeV with the ATLAS detector, Phys. Lett. B 754 (2016) 302 [arXiv: 1512.01530] [INSPIRE].

[27] ATLAS collaboration, Search for new phenomena in dijet events using $37 \mathrm{fb}^{-1}$ of $p p$ collision data collected at $\sqrt{s}=13$ TeV with the ATLAS detector, Phys. Rev. D 96 (2017) 052004 [arXiv: 1703.09127] [INSPIRE].

[28] CMS collaboration, Search for dijet resonances in 7 TeV pp collisions at CMS, Phys. Rev. Lett. 105 (2010) 211801 [arXiv:1010.0203] [INSPIRE].

[29] CMS collaboration, Search for resonances in the dijet mass spectrum from 7 TeV pp collisions at CMS, Phys. Lett. B 704 (2011) 123 [arXiv:1107.4771] [InSPIRE].

[30] CMS collaboration, Search for narrow resonances and quantum black holes in inclusive and b-tagged dijet mass spectra from pp collisions at $\sqrt{s}=7$ TeV, JHEP 01 (2013) 013 [arXiv: 1210.2387] [INSPIRE].

[31] CMS collaboration, Search for narrow resonances using the dijet mass spectrum in $p p$ collisions at $\sqrt{s}=8 \mathrm{TeV}$, Phys. Rev. D 87 (2013) 114015 [arXiv:1302.4794] [INSPIRE].

[32] CMS collaboration, Search for resonances and quantum black holes using dijet mass spectra in proton-proton collisions at $\sqrt{s}=8$ TeV, Phys. Rev. D 91 (2015) 052009 [arXiv: 1501.04198] [INSPIRE].

[33] CMS collaboration, Search for narrow resonances decaying to dijets in proton-proton collisions at $\sqrt{s}=13$ TeV, Phys. Rev. Lett. 116 (2016) 071801 [arXiv:1512.01224] [INSPIRE].

[34] CMS collaboration, Search for narrow resonances in dijet final states at $\sqrt{s}=8$ TeV with the novel CMS technique of data scouting, Phys. Rev. Lett. 117 (2016) 031802 [arXiv: 1604.08907] [INSPIRE]. 
[35] CMS collaboration, Search for dijet resonances in proton-proton collisions at $\sqrt{s}=13$ TeV and constraints on dark matter and other models, Phys. Lett. B 769 (2017) 520 [Erratum ibid. B 772 (2017) 882] [arXiv:1611.03568] [INSPIRE].

[36] CMS collaboration, Search for low mass vector resonances decaying to quark-antiquark pairs in proton-proton collisions at $\sqrt{s}=13$ TeV, Phys. Rev. Lett. 119 (2017) 111802 [arXiv: 1705.10532] [INSPIRE].

[37] B.A. Dobrescu and F. Yu, Coupling-mass mapping of dijet peak searches, Phys. Rev. D 88 (2013) 035021 [arXiv:1306.2629] [INSPIRE].

[38] D. Abercrombie et al., Dark matter benchmark models for early LHC run-2 searches: report of the ATLAS/CMS dark matter forum, arXiv:1507.00966 [INSPIRE].

[39] CMS collaboration, The CMS trigger system, 2017 JINST 12 P01020 [arXiv:1609.02366] [INSPIRE].

[40] CMS collaboration, The CMS experiment at the CERN LHC, 2008 JINST 3 S08004 [INSPIRE].

[41] J. Alwall et al., The automated computation of tree-level and next-to-leading order differential cross sections and their matching to parton shower simulations, JHEP 07 (2014) 079 [arXiv: 1405.0301] [INSPIRE].

[42] J. Alwall et al., Comparative study of various algorithms for the merging of parton showers and matrix elements in hadronic collisions, Eur. Phys. J. C 53 (2008) 473 [arXiv:0706.2569] [INSPIRE].

[43] S. Frixione, P. Nason and C. Oleari, Matching NLO QCD computations with parton shower simulations: the POWHEG method, JHEP 11 (2007) 070 [arXiv:0709.2092] [INSPIRE].

[44] T. Sjöstrand et al., An introduction to PYTHIA 8.2, Comput. Phys. Commun. 191 (2015) 159 [arXiv: 1410.3012] [INSPIRE].

[45] CMS collaboration, Event generator tunes obtained from underlying event and multiparton scattering measurements, Eur. Phys. J. C 76 (2016) 155 [arXiv:1512.00815] [INSPIRE].

[46] GEANT4 collaboration, S. Agostinelli et al., GEANT4 - a simulation toolkit, Nucl. Instrum. Meth. A 506 (2003) 250 [InSPIRE].

[47] NNPDF collaboration, R.D. Ball et al., Parton distributions for the LHC Run II, JHEP 04 (2015) 040 [arXiv: 1410.8849] [InSPIRE].

[48] CMS collaboration, Search for dark matter produced with an energetic jet or a hadronically decaying $W$ or $Z$ boson at $\sqrt{s}=13$ TeV, JHEP 07 (2017) 014 [arXiv: 1703.01651] [INSPIRE].

[49] S. Kallweit et al., NLO electroweak automation and precise predictions for $W+$ multijet production at the LHC, JHEP 04 (2015) 012 [arXiv: 1412.5157] [INSPIRE].

[50] S. Kallweit et al., NLO QCD+EW predictions for $V+$ jets including off-shell vector-boson decays and multijet merging, JHEP 04 (2016) 021 [arXiv:1511.08692] [INSPIRE].

[51] S. Kallweit, J.M. Lindert, S. Pozzorini, M. Schönherr and P. Maierhöfer, $N L O Q C D+E W$ automation and precise predictions for $V+$ multijet production, in the proceedings of the $50^{\text {th }}$ Rencontres de Moriond, QCD and high energy interactions, March 14-21, La Thuile, Italy (2015), arXiv: 1505.05704 [INSPIRE].

[52] J.M. Lindert et al., Precise predictions for $V+$ jets dark matter backgrounds, Eur. Phys. J. C 77 (2017) 829 [arXiv: 1705.04664] [INSPIRE].

[53] CMS collaboration, Particle-flow reconstruction and global event description with the CMS detector, 2017 JINST 12 P10003 [arXiv:1706.04965] [INSPIRE]. 
[54] M. Cacciari, G.P. Salam and G. Soyez, The anti- $k_{t}$ jet clustering algorithm, JHEP 04 (2008) 063 [arXiv: 0802.1189] [INSPIRE].

[55] M. Cacciari, G.P. Salam and G. Soyez, FastJet user manual, Eur. Phys. J. C 72 (2012) 1896 [arXiv: 1111.6097] [INSPIRE].

[56] D. Bertolini, P. Harris, M. Low and N. Tran, Pileup per particle identification, JHEP 10 (2014) 059 [arXiv: 1407.6013] [INSPIRE].

[57] CMS collaboration, Determination of jet energy calibration and transverse momentum resolution in CMS, 2011 JINST 6 P11002 [arXiv:1107.4277] [INSPIRE].

[58] CMS collaboration, Jet energy scale and resolution in the CMS experiment in pp collisions at $8 \mathrm{TeV}, 2017$ JINST $12 \mathrm{P} 02014$ [arXiv: 1607.03663] [INSPIRE].

[59] D. Krohn, J. Thaler and L.-T. Wang, Jet trimming, JHEP 02 (2010) 084 [arXiv: 0912.1342] [INSPIRE].

[60] M. Dasgupta, A. Fregoso, S. Marzani and G.P. Salam, Towards an understanding of jet substructure, JHEP 09 (2013) 029 [arXiv: 1307.0007] [INSPIRE].

[61] A.J. Larkoski, S. Marzani, G. Soyez and J. Thaler, Soft drop, JHEP 05 (2014) 146 [arXiv:1402.2657] [INSPIRE].

[62] J. Dolen, P. Harris, S. Marzani, S. Rappoccio and N. Tran, Thinking outside the ROCs: Designing Decorrelated Taggers (DDT) for jet substructure, JHEP 05 (2016) 156 [arXiv: 1603.00027] [INSPIRE].

[63] I. Moult, L. Necib and J. Thaler, New angles on energy correlation functions, JHEP 12 (2016) 153 [arXiv : 1609. 07483] [INSPIRE].

[64] S. A. Dudani, The distance-weighted k-nearest-neighbor rule, IEEE Trans. SMC 6 (1976) 325.

[65] CMS collaboration, Identification of b-quark jets with the CMS experiment, 2013 JINST 8 P04013 [arXiv: 1211.4462] [INSPIRE].

[66] R.A. Fisher, On the interpretation of $\chi^{2}$ from contingency tables, and the calculation of $P, J$. Roy. Stat. Soc. 85 (1922) 87.

[67] CMS collaboration, CMS luminosity measurements for the 2016 data taking period, CMS-PAS-LUM-17-001 (2017).

[68] T. Junk, Confidence level computation for combining searches with small statistics, Nucl. Instrum. Meth. A 434 (1999) 435 [hep-ex/9902006] [INSPIRE].

[69] A.L. Read, Presentation of search results: the CL(s) technique, J. Phys. G 28 (2002) 2693 [INSPIRE].

[70] G. Cowan, K. Cranmer, E. Gross and O. Vitells, Asymptotic formulae for likelihood-based tests of new physics, Eur. Phys. J. C 71 (2011) 1554 [Erratum ibid. C 73 (2013) 2501] [arXiv: 1007.1727] [INSPIRE].

[71] E. Gross and O. Vitells, Trial factors or the look elsewhere effect in high energy physics, Eur. Phys. J. C 70 (2010) 525 [arXiv: 1005.1891] [inSPIRE].

[72] B.A. Dobrescu and C. Frugiuele, Hidden GeV-scale interactions of quarks, Phys. Rev. Lett. 113 (2014) 061801 [arXiv: 1404.3947] [INSPIRE].

[73] G. Busoni et al., Recommendations on presenting LHC searches for missing transverse energy signals using simplified s-channel models of dark matter, arXiv:1603.04156 [INSPIRE].

[74] WMAP collaboration, D.N. Spergel et al., Wilkinson Microwave Anisotropy Probe (WMAP) three year results: implications for cosmology, Astrophys. J. Suppl. 170 (2007) 377 [astro-ph/0603449] [INSPIRE]. 
[75] Planck collaboration, P.A.R. Ade et al., Planck 2013 results. XVI. Cosmological parameters, Astron. Astrophys. 571 (2014) A16 [arXiv:1303.5076] [INSPIRE].

[76] M. Backovic, K. Kong and M. McCaskey, MadDM v.1.0: computation of dark matter relic abundance using MadGraph5, Physics of the Dark Universe 5-6 (2014) 18 [arXiv: 1308.4955] [INSPIRE].

[77] M. Backović et al., Direct detection of dark matter with MadDM v.2.0, Phys. Dark Univ. 9-10 (2015) 37 [arXiv: 1505.04190] [InSPIRE].

[78] T. du Pree, K. Hahn, P. Harris and C. Roskas, Cosmological constraints on Dark Matter models for collider searches, arXiv:1603.08525 [INSPIRE]. 


\section{The CMS collaboration}

\section{Yerevan Physics Institute, Yerevan, Armenia}

A.M. Sirunyan, A. Tumasyan

\section{Institut für Hochenergiephysik, Wien, Austria}

W. Adam, F. Ambrogi, E. Asilar, T. Bergauer, J. Brandstetter, E. Brondolin, M. Dragicevic, J. Erö, M. Flechl, M. Friedl, R. Frühwirth ${ }^{1}$, V.M. Ghete, J. Grossmann, J. Hrubec, M. Jeitler ${ }^{1}$, A. König, N. Krammer, I. Krätschmer, D. Liko, T. Madlener, I. Mikulec, E. Pree, N. Rad, H. Rohringer, J. Schieck ${ }^{1}$, R. Schöfbeck, M. Spanring, D. Spitzbart, W. Waltenberger, J. Wittmann, C.-E. Wulz ${ }^{1}$, M. Zarucki

Institute for Nuclear Problems, Minsk, Belarus

V. Chekhovsky, V. Mossolov, J. Suarez Gonzalez

Universiteit Antwerpen, Antwerpen, Belgium

E.A. De Wolf, D. Di Croce, X. Janssen, J. Lauwers, M. Van De Klundert, H. Van Haevermaet, P. Van Mechelen, N. Van Remortel

\section{Vrije Universiteit Brussel, Brussel, Belgium}

S. Abu Zeid, F. Blekman, J. D'Hondt, I. De Bruyn, J. De Clercq, K. Deroover, G. Flouris, D. Lontkovskyi, S. Lowette, I. Marchesini, S. Moortgat, L. Moreels, Q. Python, K. Skovpen, S. Tavernier, W. Van Doninck, P. Van Mulders, I. Van Parijs

Université Libre de Bruxelles, Bruxelles, Belgium

D. Beghin, H. Brun, B. Clerbaux, G. De Lentdecker, H. Delannoy, B. Dorney, G. Fasanella, L. Favart, R. Goldouzian, A. Grebenyuk, T. Lenzi, J. Luetic, T. Maerschalk, A. Marinov, T. Seva, E. Starling, C. Vander Velde, P. Vanlaer, D. Vannerom, R. Yonamine, F. Zenoni, F. Zhang ${ }^{2}$

\section{Ghent University, Ghent, Belgium}

A. Cimmino, T. Cornelis, D. Dobur, A. Fagot, M. Gul, I. Khvastunov ${ }^{3}$, D. Poyraz, C. Roskas, S. Salva, M. Tytgat, W. Verbeke, N. Zaganidis

\section{Université Catholique de Louvain, Louvain-la-Neuve, Belgium}

H. Bakhshiansohi, O. Bondu, S. Brochet, G. Bruno, C. Caputo, A. Caudron, P. David, S. De Visscher, C. Delaere, M. Delcourt, B. Francois, A. Giammanco, M. Komm, G. Krintiras, V. Lemaitre, A. Magitteri, A. Mertens, M. Musich, K. Piotrzkowski, L. Quertenmont, A. Saggio, M. Vidal Marono, S. Wertz, J. Zobec

\section{Centro Brasileiro de Pesquisas Fisicas, Rio de Janeiro, Brazil}

W.L. Aldá Júnior, F.L. Alves, G.A. Alves, L. Brito, M. Correa Martins Junior, C. Hensel, A. Moraes, M.E. Pol, P. Rebello Teles

Universidade do Estado do Rio de Janeiro, Rio de Janeiro, Brazil

E. Belchior Batista Das Chagas, W. Carvalho, J. Chinellato ${ }^{4}$, E. Coelho, E.M. Da Costa, G.G. Da Silveira ${ }^{5}$, D. De Jesus Damiao, S. Fonseca De Souza, L.M. Huertas Guativa, H. Malbouisson, M. Melo De Almeida, C. Mora Herrera, L. Mundim, H. Nogima, 
L.J. Sanchez Rosas, A. Santoro, A. Sznajder, M. Thiel, E.J. Tonelli Manganote ${ }^{4}$, F. Torres Da Silva De Araujo, A. Vilela Pereira

Universidade Estadual Paulista $^{a}$, Universidade Federal do ABC ${ }^{b}$, São Paulo, Brazil

S. Ahuja $^{a}$, C.A. Bernardes ${ }^{a}$, T.R. Fernandez Perez Tomei ${ }^{a}$, E.M. Gregores ${ }^{b}$, P.G. Mercadante ${ }^{b}$, S.F. Novaes ${ }^{a}$, Sandra S. Padula ${ }^{a}$, D. Romero $\operatorname{Abad}^{b}$, J.C. Ruiz Vargas ${ }^{a}$

Institute for Nuclear Research and Nuclear Energy of Bulgaria Academy of Sciences

A. Aleksandrov, R. Hadjiiska, P. Iaydjiev, M. Misheva, M. Rodozov, M. Shopova, G. Sultanov

University of Sofia, Sofia, Bulgaria

A. Dimitrov, L. Litov, B. Pavlov, P. Petkov

Beihang University, Beijing, China

W. Fang ${ }^{6}$, X. Gao ${ }^{6}$, L. Yuan

Institute of High Energy Physics, Beijing, China

M. Ahmad, J.G. Bian, G.M. Chen, H.S. Chen, M. Chen, Y. Chen, C.H. Jiang, D. Leggat, H. Liao, Z. Liu, F. Romeo, S.M. Shaheen, A. Spiezia, J. Tao, C. Wang, Z. Wang, E. Yazgan, H. Zhang, S. Zhang, J. Zhao

State Key Laboratory of Nuclear Physics and Technology, Peking University, Beijing, China

Y. Ban, G. Chen, J. Li, Q. Li, S. Liu, Y. Mao, S.J. Qian, D. Wang, Z. Xu

Universidad de Los Andes, Bogota, Colombia

C. Avila, A. Cabrera, L.F. Chaparro Sierra, C. Florez, C.F. González Hernández, J.D. Ruiz Alvarez, M.A. Segura Delgado

University of Split, Faculty of Electrical Engineering, Mechanical Engineering and Naval Architecture, Split, Croatia

B. Courbon, N. Godinovic, D. Lelas, I. Puljak, P.M. Ribeiro Cipriano, T. Sculac

University of Split, Faculty of Science, Split, Croatia

Z. Antunovic, M. Kovac

Institute Rudjer Boskovic, Zagreb, Croatia

V. Brigljevic, D. Ferencek, K. Kadija, B. Mesic, A. Starodumov ${ }^{7}$, T. Susa

University of Cyprus, Nicosia, Cyprus

M.W. Ather, A. Attikis, G. Mavromanolakis, J. Mousa, C. Nicolaou, F. Ptochos, P.A. Razis, H. Rykaczewski

Charles University, Prague, Czech Republic

M. Finger ${ }^{8}$, M. Finger Jr. ${ }^{8}$

Universidad San Francisco de Quito, Quito, Ecuador

E. Carrera Jarrin 
Academy of Scientific Research and Technology of the Arab Republic of Egypt, Egyptian Network of High Energy Physics, Cairo, Egypt

E. El-khateeb ${ }^{9}$, S. Elgammal ${ }^{10}$, A. Ellithi Kamel ${ }^{11}$

National Institute of Chemical Physics and Biophysics, Tallinn, Estonia

R.K. Dewanjee, M. Kadastik, L. Perrini, M. Raidal, A. Tiko, C. Veelken

Department of Physics, University of Helsinki, Helsinki, Finland

P. Eerola, H. Kirschenmann, J. Pekkanen, M. Voutilainen

Helsinki Institute of Physics, Helsinki, Finland

J. Havukainen, J.K. Heikkilä, T. Järvinen, V. Karimäki, R. Kinnunen, T. Lampén, K. Lassila-Perini, S. Laurila, S. Lehti, T. Lindén, P. Luukka, H. Siikonen, E. Tuominen, J. Tuominiemi

Lappeenranta University of Technology, Lappeenranta, Finland

T. Tuuva

IRFU, CEA, Université Paris-Saclay, Gif-sur-Yvette, France

M. Besancon, F. Couderc, M. Dejardin, D. Denegri, J.L. Faure, F. Ferri, S. Ganjour, S. Ghosh, P. Gras, G. Hamel de Monchenault, P. Jarry, I. Kucher, C. Leloup, E. Locci, M. Machet, J. Malcles, G. Negro, J. Rander, A. Rosowsky, M.Ö. Sahin, M. Titov

Laboratoire Leprince-Ringuet, Ecole polytechnique, CNRS/IN2P3, Université Paris-Saclay, Palaiseau, France

A. Abdulsalam, C. Amendola, I. Antropov, S. Baffioni, F. Beaudette, P. Busson, L. Cadamuro, C. Charlot, R. Granier de Cassagnac, M. Jo, S. Lisniak, A. Lobanov, J. Martin Blanco, M. Nguyen, C. Ochando, G. Ortona, P. Paganini, P. Pigard, R. Salerno, J.B. Sauvan, Y. Sirois, A.G. Stahl Leiton, T. Strebler, Y. Yilmaz, A. Zabi, A. Zghiche

Université de Strasbourg, CNRS, IPHC UMR 7178, F-67000 Strasbourg, France

J.-L. Agram ${ }^{12}$, J. Andrea, D. Bloch, J.-M. Brom, M. Buttignol, E.C. Chabert, N. Chanon, C. Collard, E. Conte ${ }^{12}$, X. Coubez, J.-C. Fontaine ${ }^{12}$, D. Gelé, U. Goerlach, M. Jansová, A.-C. Le Bihan, N. Tonon, P. Van Hove

Centre de Calcul de l'Institut National de Physique Nucleaire et de Physique des Particules, CNRS/IN2P3, Villeurbanne, France

S. Gadrat

Université de Lyon, Université Claude Bernard Lyon 1, CNRS-IN2P3, Institut de Physique Nucléaire de Lyon, Villeurbanne, France

S. Beauceron, C. Bernet, G. Boudoul, R. Chierici, D. Contardo, P. Depasse, H. El Mamouni, J. Fay, L. Finco, S. Gascon, M. Gouzevitch, G. Grenier, B. Ille, F. Lagarde, I.B. Laktineh, M. Lethuillier, L. Mirabito, A.L. Pequegnot, S. Perries, A. Popov ${ }^{13}$, V. Sordini, M. Vander Donckt, S. Viret

Georgian Technical University, Tbilisi, Georgia

T. Toriashvili ${ }^{14}$ 
Tbilisi State University, Tbilisi, Georgia

Z. Tsamalaidze ${ }^{8}$

RWTH Aachen University, I. Physikalisches Institut, Aachen, Germany

C. Autermann, L. Feld, M.K. Kiesel, K. Klein, M. Lipinski, M. Preuten, C. Schomakers, J. Schulz, V. Zhukov ${ }^{13}$

RWTH Aachen University, III. Physikalisches Institut A, Aachen, Germany

A. Albert, E. Dietz-Laursonn, D. Duchardt, M. Endres, M. Erdmann, S. Erdweg, T. Esch, R. Fischer, A. Güth, M. Hamer, T. Hebbeker, C. Heidemann, K. Hoepfner, S. Knutzen, M. Merschmeyer, A. Meyer, P. Millet, S. Mukherjee, T. Pook, M. Radziej, H. Reithler, M. Rieger, F. Scheuch, D. Teyssier, S. Thüer

RWTH Aachen University, III. Physikalisches Institut B, Aachen, Germany

G. Flügge, B. Kargoll, T. Kress, A. Künsken, T. Müller, A. Nehrkorn, A. Nowack, C. Pistone, O. Pooth, A. Stahl ${ }^{15}$

\section{Deutsches Elektronen-Synchrotron, Hamburg, Germany}

M. Aldaya Martin, T. Arndt, C. Asawatangtrakuldee, K. Beernaert, O. Behnke, U. Behrens, A. Bermúdez Martínez, A.A. Bin Anuar, K. Borras ${ }^{16}$, V. Botta, A. Campbell, P. Connor, C. Contreras-Campana, F. Costanza, C. Diez Pardos, G. Eckerlin, D. Eckstein, T. Eichhorn, E. Eren, E. Gallo ${ }^{17}$, J. Garay Garcia, A. Geiser, J.M. Grados Luyando, A. Grohsjean, P. Gunnellini, M. Guthoff, A. Harb, J. Hauk, M. Hempel ${ }^{18}$, H. Jung, M. Kasemann, J. Keaveney, C. Kleinwort, I. Korol, D. Krücker, W. Lange, A. Lelek, T. Lenz, J. Leonard, K. Lipka, W. Lohmann ${ }^{18}$, R. Mankel, I.-A. Melzer-Pellmann, A.B. Meyer, G. Mittag, J. Mnich, A. Mussgiller, E. Ntomari, D. Pitzl, A. Raspereza, M. Savitskyi, P. Saxena, R. Shevchenko, S. Spannagel, N. Stefaniuk, G.P. Van Onsem, R. Walsh, Y. Wen, K. Wichmann, C. Wissing, O. Zenaiev

\section{University of Hamburg, Hamburg, Germany}

R. Aggleton, S. Bein, V. Blobel, M. Centis Vignali, T. Dreyer, E. Garutti, D. Gonzalez, J. Haller, A. Hinzmann, M. Hoffmann, A. Karavdina, R. Klanner, R. Kogler, N. Kovalchuk, S. Kurz, T. Lapsien, D. Marconi, M. Meyer, M. Niedziela, D. Nowatschin, F. Pantaleo ${ }^{15}$, T. Peiffer, A. Perieanu, C. Scharf, P. Schleper, A. Schmidt, S. Schumann, J. Schwandt, J. Sonneveld, H. Stadie, G. Steinbrück, F.M. Stober, M. Stöver, H. Tholen, D. Troendle, E. Usai, A. Vanhoefer, B. Vormwald

\section{Institut für Experimentelle Kernphysik, Karlsruhe, Germany}

M. Akbiyik, C. Barth, M. Baselga, S. Baur, E. Butz, R. Caspart, T. Chwalek, F. Colombo, W. De Boer, A. Dierlamm, N. Faltermann, B. Freund, R. Friese, M. Giffels, M.A. Harrendorf, F. Hartmann ${ }^{15}$, S.M. Heindl, U. Husemann, F. Kassel ${ }^{15}$, S. Kudella, H. Mildner, M.U. Mozer, Th. Müller, M. Plagge, G. Quast, K. Rabbertz, M. Schröder, I. Shvetsov, G. Sieber, H.J. Simonis, R. Ulrich, S. Wayand, M. Weber, T. Weiler, S. Williamson, C. Wöhrmann, R. Wolf 
Institute of Nuclear and Particle Physics (INPP), NCSR Demokritos, Aghia Paraskevi, Greece

G. Anagnostou, G. Daskalakis, T. Geralis, A. Kyriakis, D. Loukas, I. Topsis-Giotis

National and Kapodistrian University of Athens, Athens, Greece

G. Karathanasis, S. Kesisoglou, A. Panagiotou, N. Saoulidou

National Technical University of Athens, Athens, Greece

K. Kousouris

University of Ioánnina, Ioánnina, Greece

I. Evangelou, C. Foudas, P. Gianneios, P. Katsoulis, P. Kokkas, S. Mallios, N. Manthos,

I. Papadopoulos, E. Paradas, J. Strologas, F.A. Triantis, D. Tsitsonis

MTA-ELTE Lendület CMS Particle and Nuclear Physics Group, Eötvös Loránd University, Budapest, Hungary

M. Csanad, N. Filipovic, G. Pasztor, O. Surányi, G.I. Veres ${ }^{19}$

Wigner Research Centre for Physics, Budapest, Hungary

G. Bencze, C. Hajdu, D. Horvath ${ }^{20}$, Á. Hunyadi, F. Sikler, V. Veszpremi

Institute of Nuclear Research ATOMKI, Debrecen, Hungary

N. Beni, S. Czellar, J. Karancsi ${ }^{21}$, A. Makovec, J. Molnar, Z. Szillasi

Institute of Physics, University of Debrecen, Debrecen, Hungary

M. Bartók ${ }^{19}$, P. Raics, Z.L. Trocsanyi, B. Ujvari

Indian Institute of Science (IISc), Bangalore, India

S. Choudhury, J.R. Komaragiri

National Institute of Science Education and Research, Bhubaneswar, India

S. Bahinipati ${ }^{22}$, S. Bhowmik, P. Mal, K. Mandal, A. Nayak ${ }^{23}$, D.K. Sahoo ${ }^{22}$, N. Sahoo, S.K. Swain

Panjab University, Chandigarh, India

S. Bansal, S.B. Beri, V. Bhatnagar, R. Chawla, N. Dhingra, A.K. Kalsi, A. Kaur, M. Kaur, S. Kaur, R. Kumar, P. Kumari, A. Mehta, J.B. Singh, G. Walia

University of Delhi, Delhi, India

Ashok Kumar, Aashaq Shah, A. Bhardwaj, S. Chauhan, B.C. Choudhary, R.B. Garg, S. Keshri, A. Kumar, S. Malhotra, M. Naimuddin, K. Ranjan, R. Sharma

Saha Institute of Nuclear Physics, HBNI, Kolkata, India

R. Bhardwaj, R. Bhattacharya, S. Bhattacharya, U. Bhawandeep, S. Dey, S. Dutt, S. Dutta, S. Ghosh, N. Majumdar, A. Modak, K. Mondal, S. Mukhopadhyay, S. Nandan, A. Purohit, A. Roy, S. Roy Chowdhury, S. Sarkar, M. Sharan, S. Thakur

Indian Institute of Technology Madras, Madras, India P.K. Behera 


\section{Bhabha Atomic Research Centre, Mumbai, India}

R. Chudasama, D. Dutta, V. Jha, V. Kumar, A.K. Mohanty ${ }^{15}$, P.K. Netrakanti, L.M. Pant, P. Shukla, A. Topkar

Tata Institute of Fundamental Research-A, Mumbai, India

T. Aziz, S. Dugad, B. Mahakud, S. Mitra, G.B. Mohanty, N. Sur, B. Sutar

\section{Tata Institute of Fundamental Research-B, Mumbai, India}

S. Banerjee, S. Bhattacharya, S. Chatterjee, P. Das, M. Guchait, Sa. Jain, S. Kumar, M. Maity ${ }^{24}$, G. Majumder, K. Mazumdar, T. Sarkar ${ }^{24}$, N. Wickramage ${ }^{25}$

Indian Institute of Science Education and Research (IISER), Pune, India

S. Chauhan, S. Dube, V. Hegde, A. Kapoor, K. Kothekar, S. Pandey, A. Rane, S. Sharma

Institute for Research in Fundamental Sciences (IPM), Tehran, Iran

S. Chenarani ${ }^{26}$, E. Eskandari Tadavani, S.M. Etesami ${ }^{26}$, M. Khakzad, M. Mohammadi Najafabadi, M. Naseri, S. Paktinat Mehdiabadi ${ }^{27}$, F. Rezaei Hosseinabadi, B. Safarzadeh ${ }^{28}$, M. Zeinali

\section{University College Dublin, Dublin, Ireland}

M. Felcini, M. Grunewald

INFN Sezione di Bari ${ }^{a}$, Università di Bari ${ }^{b}$, Politecnico di Bari ${ }^{c}$, Bari, Italy M. Abbrescia ${ }^{a, b}$, C. Calabria ${ }^{a, b}$, A. Colaleo ${ }^{a}$, D. Creanza ${ }^{a, c}$, L. Cristella ${ }^{a, b}$, N. De

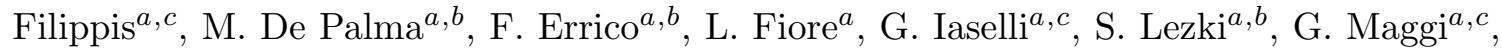
M. Maggi ${ }^{a}$, G. Miniello ${ }^{a, b}$, S. My ${ }^{a, b}$, S. Nuzzo ${ }^{a, b}$, A. Pompili ${ }^{a, b}$, G. Pugliese ${ }^{a, c}$, R. Radogna ${ }^{a}$, A. Ranieri ${ }^{a}$, G. Selvaggi ${ }^{a, b}$, A. Sharma ${ }^{a}$, L. Silvestris ${ }^{a, 15}$, R. Venditti ${ }^{a}$, P. Verwilligen ${ }^{a}$

INFN Sezione di Bologna ${ }^{a}$, Università di Bologna ${ }^{b}$, Bologna, Italy

G. Abbiendi ${ }^{a}$, C. Battilana ${ }^{a, b}$, D. Bonacorsi ${ }^{a, b}$, L. Borgonovi $^{a, b}$, S. Braibant-Giacomelli ${ }^{a, b}$, R. Campanini ${ }^{a, b}$, P. Capiluppi ${ }^{a, b}$, A. Castro ${ }^{a, b}$, F.R. Cavallo ${ }^{a}$, S.S. Chhibra ${ }^{a}$, G. Codispoti ${ }^{a, b}$, M. Cuffiani ${ }^{a, b}$, G.M. Dallavalle ${ }^{a}$, F. Fabbri $^{a}$, A. Fanfani ${ }^{a, b}$, D. Fasanella ${ }^{a, b}$, P. Giacomelli ${ }^{a}$, C. Grandi ${ }^{a}$, L. Guiducci ${ }^{a}, b$, S. Marcellini ${ }^{a}$, G. Masetti ${ }^{a}$, A. Montanari ${ }^{a}$,

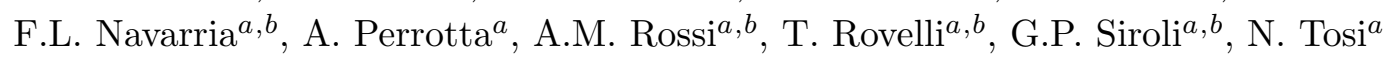

INFN Sezione di Catania ${ }^{a}$, Università di Catania ${ }^{b}$, Catania, Italy

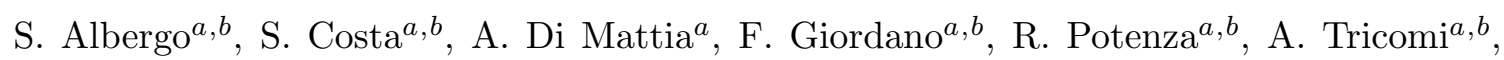
C. Tuve $\mathrm{e}^{a, b}$

INFN Sezione di Firenze ${ }^{a}$, Università di Firenze ${ }^{b}$, Firenze, Italy

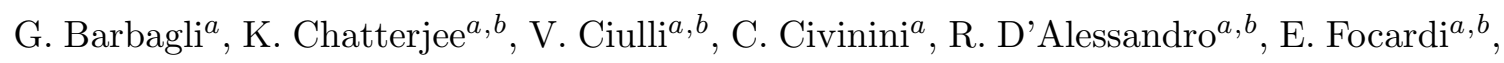

P. Lenzi ${ }^{a, b}$, M. Meschini ${ }^{a}$, S. Paoletti ${ }^{a}$, L. Russo ${ }^{a, 29}$, G. Sguazzoni ${ }^{a}$, D. Strom ${ }^{a}$, L. Viliani ${ }^{a, b, 15}$

INFN Laboratori Nazionali di Frascati, Frascati, Italy

L. Benussi, S. Bianco, F. Fabbri, D. Piccolo, F. Primavera ${ }^{15}$ 
INFN Sezione di Genova ${ }^{a}$, Università di Genova ${ }^{b}$, Genova, Italy V. Calvelli ${ }^{a, b}$, F. Ferro ${ }^{a}$, E. Robutti ${ }^{a}$, S. Tosi ${ }^{a, b}$

INFN Sezione di Milano-Bicocca ${ }^{a}$, Università di Milano-Bicocca ${ }^{b}$, Milano, Italy

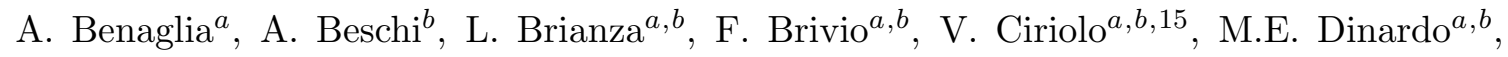
S. Fiorendi ${ }^{a, b}$, S. Gennai ${ }^{a}$, A. Ghezzi ${ }^{a, b}$, P. Govoni ${ }^{a, b}$, M. Malberti ${ }^{a, b}$, S. Malvezzi ${ }^{a}$, R.A. Manzoni ${ }^{a, b}$, D. Menasce ${ }^{a}$, L. Moroni $^{a}$, M. Paganoni ${ }^{a, b}$, K. Pauwels $^{a, b}$, D. Pedrini ${ }^{a}$, S. Pigazzini ${ }^{a, b, 30}$, S. Ragazzi ${ }^{a, b}$, T. Tabarelli de Fatis ${ }^{a, b}$

INFN Sezione di Napoli ${ }^{a}$, Università di Napoli 'Federico II' ${ }^{b}$, Napoli, Italy, Università della Basilicata ${ }^{c}$, Potenza, Italy, Università G. Marconi ${ }^{d}$, Roma, Italy

S. Buontempo ${ }^{a}$, N. Cavallo ${ }^{a, c}$, S. Di Guida ${ }^{a, d, 15}$, F. Fabozzi ${ }^{a, c}$, F. Fienga ${ }^{a, b}$,

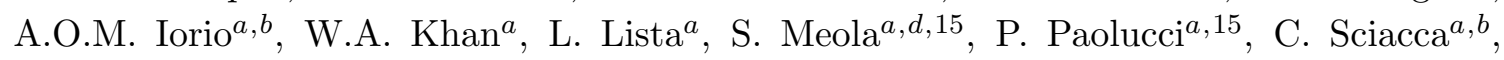
F. Thyssen ${ }^{a}$

INFN Sezione di Padova ${ }^{a}$, Università di Padova ${ }^{b}$, Padova, Italy, Università di Trento ${ }^{c}$, Trento, Italy

P. Azzi ${ }^{a}$, N. Bacchetta ${ }^{a}$, L. Benato ${ }^{a, b}$, D. Bisello ${ }^{a, b}$, A. Boletti ${ }^{a, b}$, A. Carvalho Antunes De Oliveira $^{a, b}$, P. Checchia ${ }^{a}$, M. Dall'Osso ${ }^{a, b}$, P. De Castro Manzano ${ }^{a}$, T. Dorigo ${ }^{a}$, U. Dosselli ${ }^{a}$, F. Gasparini ${ }^{a}, b$, A. Gozzelino ${ }^{a}$, S. Lacaprara ${ }^{a}$, P. Lujan, M. Margoni ${ }^{a, b}$, $^{a}$ A.T. Meneguzzo ${ }^{a, b}$, D. Pantano ${ }^{a}$, N. Pozzobon ${ }^{a, b}$, P. Ronchese ${ }^{a, b}$, R. $\operatorname{Rossin}^{a, b}$, E. Torassa ${ }^{a}$, S. Ventura ${ }^{a}$, M. Zanetti ${ }^{a, b}$, P. Zotto $^{a, b}$, G. Zumerle ${ }^{a, b}$

INFN Sezione di Pavia ${ }^{a}$, Università di Pavia ${ }^{b}$, Pavia, Italy

A. Braghieri ${ }^{a}$, A. Magnani ${ }^{a}$, P. Montagna ${ }^{a, b}$, S.P. Ratti ${ }^{a}, b$, V. $\operatorname{Re}^{a}$, M. Ressegotti ${ }^{a, b}$, C. Riccardi ${ }^{a, b}$, P. Salvini ${ }^{a}$, I. Vai ${ }^{a, b}$, P. Vitulo ${ }^{a, b}$

INFN Sezione di Perugia ${ }^{a}$, Università di Perugia ${ }^{b}$, Perugia, Italy

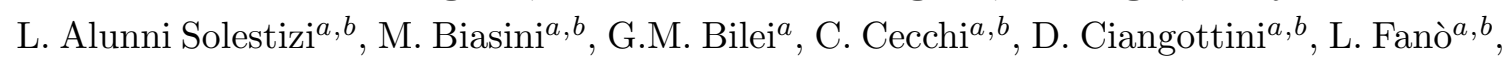

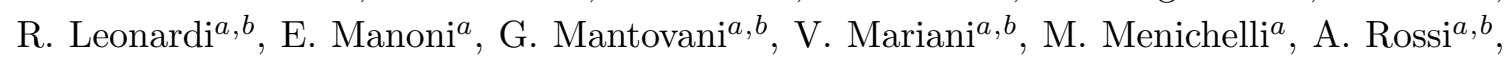
A. Santocchia ${ }^{a, b}$, D. Spiga ${ }^{a}$

INFN Sezione di Pisa ${ }^{a}$, Università di Pisa ${ }^{b}$, Scuola Normale Superiore di Pisa ${ }^{c}$, Pisa, Italy

K. Androsov ${ }^{a}$, P. Azzurri ${ }^{a, 15}$, G. Bagliesi ${ }^{a}$, T. Boccali ${ }^{a}$, L. Borrello, R. Castaldi ${ }^{a}$, M.A. Ciocci ${ }^{a}, b$, R. Dell'Orso ${ }^{a}$, G. Fedi ${ }^{a}$, L. Giannini ${ }^{a, c}$, A. Giassi ${ }^{a}$, M.T. Grippo ${ }^{a, 29}$, F. Ligabue ${ }^{a, c}$, T. Lomtadze $^{a}$, E. Manca ${ }^{a, c}$, G. Mandorli ${ }^{a, c}$, A. Messineo ${ }^{a, b}$, F. Palla ${ }^{a}$,

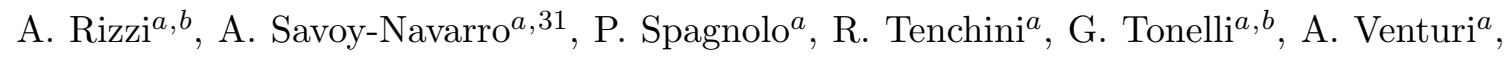
P.G. Verdini ${ }^{a}$

INFN Sezione di Roma ${ }^{a}$, Sapienza Università di Roma ${ }^{b}$, Rome, Italy

L. Barone ${ }^{a, b}$, F. Cavallari ${ }^{a}$, M. Cipriani ${ }^{a, b}$, N. Daci ${ }^{a}$, D. Del Re $e^{a, b, 15}$, E. Di

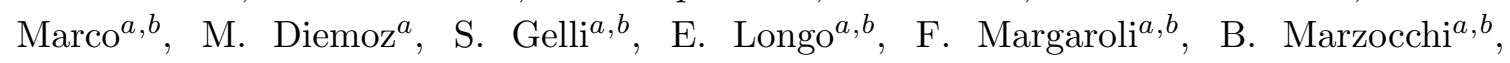
P. Meridiani ${ }^{a}$, G. Organtini ${ }^{a, b}$, R. Paramatti ${ }^{a}, b$, F. Preiato ${ }^{a, b}$, S. Rahatlou $^{a, b}$, C. Rovelli $^{a}$, F. Santanastasio ${ }^{a, b}$ 
INFN Sezione di Torino ${ }^{a}$, Università di Torino ${ }^{b}$, Torino, Italy, Università del Piemonte Orientale ${ }^{c}$, Novara, Italy

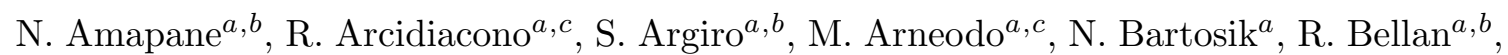
C. Biino ${ }^{a}$, N. Cartiglia ${ }^{a}$, F. Cenna ${ }^{a, b}$, M. Costa ${ }^{a, b}$, R. Covarelli ${ }^{a, b}$, A. Degano ${ }^{a, b}$, N. Demaria ${ }^{a}$, B. Kiani ${ }^{a, b}$, C. Mariotti ${ }^{a}$, S. Maselli ${ }^{a}$, E. Migliore ${ }^{a, b}$, V. Monaco ${ }^{a, b}$,

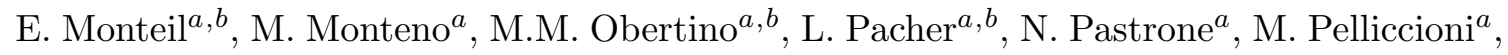
G.L. Pinna Angioni ${ }^{a, b}$, F. Ravera ${ }^{a, b}$, A. Romero ${ }^{a, b}$, M. Ruspa ${ }^{a, c}$, R. Sacchi ${ }^{a, b}$, K. Shchelina ${ }^{a, b}$, V. Sola ${ }^{a}$, A. Solano ${ }^{a, b}$, A. Staiano ${ }^{a}$, P. Traczyk ${ }^{a, b}$

INFN Sezione di Trieste ${ }^{a}$, Università di Trieste ${ }^{b}$, Trieste, Italy

S. Belforte ${ }^{a}$, M. Casarsa ${ }^{a}$, F. Cossutti ${ }^{a}$, G. Della Ricca ${ }^{a}, b$, A. Zanetti ${ }^{a}$

Kyungpook National University, Daegu, Korea

D.H. Kim, G.N. Kim, M.S. Kim, J. Lee, S. Lee, S.W. Lee, C.S. Moon, Y.D. Oh, S. Sekmen, D.C. Son, Y.C. Yang

Chonbuk National University, Jeonju, Korea

A. Lee

Chonnam National University, Institute for Universe and Elementary Particles, Kwangju, Korea

H. Kim, D.H. Moon, G. Oh

Hanyang University, Seoul, Korea

J.A. Brochero Cifuentes, J. Goh, T.J. Kim

Korea University, Seoul, Korea

S. Cho, S. Choi, Y. Go, D. Gyun, S. Ha, B. Hong, Y. Jo, Y. Kim, K. Lee, K.S. Lee, S. Lee, J. Lim, S.K. Park, Y. Roh

Seoul National University, Seoul, Korea

J. Almond, J. Kim, J.S. Kim, H. Lee, K. Lee, K. Nam, S.B. Oh, B.C. Radburn-Smith, S.h. Seo, U.K. Yang, H.D. Yoo, G.B. Yu

University of Seoul, Seoul, Korea

H. Kim, J.H. Kim, J.S.H. Lee, I.C. Park

Sungkyunkwan University, Suwon, Korea

Y. Choi, C. Hwang, J. Lee, I. Yu

Vilnius University, Vilnius, Lithuania

V. Dudenas, A. Juodagalvis, J. Vaitkus

National Centre for Particle Physics, Universiti Malaya, Kuala Lumpur, Malaysia

I. Ahmed, Z.A. Ibrahim, M.A.B. Md Ali ${ }^{32}$, F. Mohamad Idris ${ }^{33}$, W.A.T. Wan Abdullah, M.N. Yusli, Z. Zolkapli 
Centro de Investigacion y de Estudios Avanzados del IPN, Mexico City, Mexico Reyes-Almanza, R, Ramirez-Sanchez, G., Duran-Osuna, M. C., H. Castilla-Valdez, E. De La Cruz-Burelo, I. Heredia-De La Cruz ${ }^{34}$, Rabadan-Trejo, R. I., R. Lopez-Fernandez, J. Mejia Guisao, A. Sanchez-Hernandez

\section{Universidad Iberoamericana, Mexico City, Mexico}

S. Carrillo Moreno, C. Oropeza Barrera, F. Vazquez Valencia

Benemerita Universidad Autonoma de Puebla, Puebla, Mexico

J. Eysermans, I. Pedraza, H.A. Salazar Ibarguen, C. Uribe Estrada

Universidad Autónoma de San Luis Potosí, San Luis Potosí, Mexico

A. Morelos Pineda

University of Auckland, Auckland, New Zealand

D. Krofcheck

University of Canterbury, Christchurch, New Zealand

P.H. Butler

National Centre for Physics, Quaid-I-Azam University, Islamabad, Pakistan

A. Ahmad, M. Ahmad, Q. Hassan, H.R. Hoorani, A. Saddique, M.A. Shah, M. Shoaib, M. Waqas

National Centre for Nuclear Research, Swierk, Poland

H. Bialkowska, M. Bluj, B. Boimska, T. Frueboes, M. Górski, M. Kazana, K. Nawrocki, M. Szleper, P. Zalewski

Institute of Experimental Physics, Faculty of Physics, University of Warsaw, Warsaw, Poland

K. Bunkowski, A. Byszuk ${ }^{35}$, K. Doroba, A. Kalinowski, M. Konecki, J. Krolikowski, M. Misiura, M. Olszewski, A. Pyskir, M. Walczak

Laboratório de Instrumentação e Física Experimental de Partículas, Lisboa, Portugal

P. Bargassa, C. Beirão Da Cruz E Silva, A. Di Francesco, P. Faccioli, B. Galinhas, M. Gallinaro, J. Hollar, N. Leonardo, L. Lloret Iglesias, M.V. Nemallapudi, J. Seixas, G. Strong, O. Toldaiev, D. Vadruccio, J. Varela

Joint Institute for Nuclear Research, Dubna, Russia

S. Afanasiev, P. Bunin, M. Gavrilenko, I. Golutvin, I. Gorbunov, A. Kamenev, V. Karjavin, A. Lanev, A. Malakhov, V. Matveev ${ }^{36,37}$, V. Palichik, V. Perelygin, S. Shmatov, S. Shulha, N. Skatchkov, V. Smirnov, N. Voytishin, A. Zarubin

Petersburg Nuclear Physics Institute, Gatchina (St. Petersburg), Russia Y. Ivanov, V. Kim ${ }^{38}$, E. Kuznetsova ${ }^{39}$, P. Levchenko, V. Murzin, V. Oreshkin, I. Smirnov, D. Sosnov, V. Sulimov, L. Uvarov, S. Vavilov, A. Vorobyev 
Institute for Nuclear Research, Moscow, Russia

Yu. Andreev, A. Dermenev, S. Gninenko, N. Golubev, A. Karneyeu, M. Kirsanov, N. Krasnikov, A. Pashenkov, D. Tlisov, A. Toropin

Institute for Theoretical and Experimental Physics, Moscow, Russia

V. Epshteyn, V. Gavrilov, N. Lychkovskaya, V. Popov, I. Pozdnyakov, G. Safronov, A. Spiridonov, A. Stepennov, M. Toms, E. Vlasov, A. Zhokin

Moscow Institute of Physics and Technology, Moscow, Russia

T. Aushev, A. Bylinkin ${ }^{37}$

National Research Nuclear University 'Moscow Engineering Physics Institute' (MEPhI), Moscow, Russia

M. Chadeeva ${ }^{40}$, O. Markin, P. Parygin, D. Philippov, S. Polikarpov, V. Rusinov

P.N. Lebedev Physical Institute, Moscow, Russia

V. Andreev, M. Azarkin ${ }^{37}$, I. Dremin ${ }^{37}$, M. Kirakosyan ${ }^{37}$, A. Terkulov

Skobeltsyn Institute of Nuclear Physics, Lomonosov Moscow State University, Moscow, Russia

A. Baskakov, A. Belyaev, E. Boos, V. Bunichev, M. Dubinin ${ }^{41}$, L. Dudko, A. Gribushin, V. Klyukhin, O. Kodolova, I. Lokhtin, I. Miagkov, S. Obraztsov, S. Petrushanko, V. Savrin, A. Snigirev

Novosibirsk State University (NSU), Novosibirsk, Russia

V. Blinov ${ }^{42}$, Y.Skovpen $^{42}$, D. Shtol ${ }^{42}$

State Research Center of Russian Federation, Institute for High Energy Physics, Protvino, Russia

I. Azhgirey, I. Bayshev, S. Bitioukov, D. Elumakhov, A. Godizov, V. Kachanov, A. Kalinin, D. Konstantinov, P. Mandrik, V. Petrov, R. Ryutin, A. Sobol, S. Troshin, N. Tyurin, A. Uzunian, A. Volkov

University of Belgrade, Faculty of Physics and Vinca Institute of Nuclear Sciences, Belgrade, Serbia

P. Adzic ${ }^{43}$, P. Cirkovic, D. Devetak, M. Dordevic, J. Milosevic, V. Rekovic

Centro de Investigaciones Energéticas Medioambientales y Tecnológicas (CIEMAT), Madrid, Spain

J. Alcaraz Maestre, I. Bachiller, M. Barrio Luna, M. Cerrada, N. Colino, B. De La Cruz, A. Delgado Peris, A. Escalante Del Valle, C. Fernandez Bedoya, J.P. Fernández Ramos, J. Flix, M.C. Fouz, O. Gonzalez Lopez, S. Goy Lopez, J.M. Hernandez, M.I. Josa, D. Moran, A. Pérez-Calero Yzquierdo, J. Puerta Pelayo, A. Quintario Olmeda, I. Redondo,

L. Romero, M.S. Soares, A. Álvarez Fernández

Universidad Autónoma de Madrid, Madrid, Spain

C. Albajar, J.F. de Trocóniz, M. Missiroli 


\section{Universidad de Oviedo, Oviedo, Spain}

J. Cuevas, C. Erice, J. Fernandez Menendez, I. Gonzalez Caballero, J.R. González Fernández, E. Palencia Cortezon, S. Sanchez Cruz, P. Vischia, J.M. Vizan Garcia

Instituto de Física de Cantabria (IFCA), CSIC-Universidad de Cantabria, Santander, Spain

I.J. Cabrillo, A. Calderon, B. Chazin Quero, E. Curras, J. Duarte Campderros, M. Fernandez, J. Garcia-Ferrero, G. Gomez, A. Lopez Virto, J. Marco, C. Martinez Rivero, P. Martinez Ruiz del Arbol, F. Matorras, J. Piedra Gomez, T. Rodrigo, A. Ruiz-Jimeno, L. Scodellaro, N. Trevisani, I. Vila, R. Vilar Cortabitarte

\section{CERN, European Organization for Nuclear Research, Geneva, Switzerland}

D. Abbaneo, B. Akgun, E. Auffray, P. Baillon, A.H. Ball, D. Barney, J. Bendavid, M. Bianco, P. Bloch, A. Bocci, C. Botta, T. Camporesi, R. Castello, M. Cepeda, G. Cerminara, E. Chapon, Y. Chen, D. d'Enterria, A. Dabrowski, V. Daponte, A. David, M. De Gruttola, A. De Roeck, N. Deelen, M. Dobson, T. du Pree, M. Dünser, N. Dupont, A. Elliott-Peisert, P. Everaerts, F. Fallavollita, G. Franzoni, J. Fulcher, W. Funk, D. Gigi, A. Gilbert, K. Gill, F. Glege, D. Gulhan, P. Harris, J. Hegeman, V. Innocente, A. Jafari, P. Janot, O. Karacheban ${ }^{18}$, J. Kieseler, V. Knünz, A. Kornmayer, M.J. Kortelainen, M. Krammer ${ }^{1}$, C. Lange, P. Lecoq, C. Lourenço, M.T. Lucchini, L. Malgeri, M. Mannelli, A. Martelli, F. Meijers, J.A. Merlin, S. Mersi, E. Meschi, P. Milenovic ${ }^{44}$, F. Moortgat, M. Mulders, H. Neugebauer, J. Ngadiuba, S. Orfanelli, L. Orsini, L. Pape, E. Perez, M. Peruzzi, A. Petrilli, G. Petrucciani, A. Pfeiffer, M. Pierini, D. Rabady, A. Racz, T. Reis,

G. Rolandi ${ }^{45}$, M. Rovere, H. Sakulin, C. Schäfer, C. Schwick, M. Seidel, M. Selvaggi, A. Sharma, P. Silva, P. Sphicas ${ }^{46}$, A. Stakia, J. Steggemann, M. Stoye, M. Tosi, D. Treille, A. Triossi, A. Tsirou, V. Veckalns ${ }^{47}$, M. Verweij, W.D. Zeuner

\section{Paul Scherrer Institut, Villigen, Switzerland}

W. Bertl ${ }^{\dagger}$, L. Caminada ${ }^{48}$, K. Deiters, W. Erdmann, R. Horisberger, Q. Ingram, H.C. Kaestli, D. Kotlinski, U. Langenegger, T. Rohe, S.A. Wiederkehr

\section{Institute for Particle Physics and Astrophysics (IPA), Zurich, Switzerland}

M. Backhaus, L. Bäni, P. Berger, L. Bianchini, B. Casal, G. Dissertori, M. Dittmar, M. Donegà, C. Dorfer, C. Grab, C. Heidegger, D. Hits, J. Hoss, G. Kasieczka, T. Klijnsma, W. Lustermann, B. Mangano, M. Marionneau, M.T. Meinhard, D. Meister, F. Micheli, P. Musella, F. Nessi-Tedaldi, F. Pandolfi, J. Pata, F. Pauss, G. Perrin, L. Perrozzi, M. Quittnat, M. Reichmann, D.A. Sanz Becerra, M. Schönenberger, L. Shchutska, V.R. Tavolaro, K. Theofilatos, M.L. Vesterbacka Olsson, R. Wallny, D.H. Zhu

\section{Universität Zürich, Zurich, Switzerland}

T.K. Aarrestad, C. Amsler ${ }^{49}$, M.F. Canelli, A. De Cosa, R. Del Burgo, S. Donato, C. Galloni, T. Hreus, B. Kilminster, D. Pinna, G. Rauco, P. Robmann, D. Salerno, K. Schweiger, C. Seitz, Y. Takahashi, A. Zucchetta 
National Central University, Chung-Li, Taiwan

V. Candelise, Y.H. Chang, K.y. Cheng, T.H. Doan, Sh. Jain, R. Khurana, C.M. Kuo,

W. Lin, A. Pozdnyakov, S.S. Yu

National Taiwan University (NTU), Taipei, Taiwan

Arun Kumar, P. Chang, Y. Chao, K.F. Chen, P.H. Chen, F. Fiori, W.-S. Hou, Y. Hsiung, Y.F. Liu, R.-S. Lu, E. Paganis, A. Psallidas, A. Steen, J.f. Tsai

Chulalongkorn University, Faculty of Science, Department of Physics, Bangkok, Thailand

B. Asavapibhop, K. Kovitanggoon, G. Singh, N. Srimanobhas

Çukurova University, Physics Department, Science and Art Faculty, Adana, Turkey

M.N. Bakirci ${ }^{50}$, A. Bat, F. Boran, S. Damarseckin, Z.S. Demiroglu, C. Dozen, E. Eskut, S. Girgis, G. Gokbulut, Y. Guler, I. Hos ${ }^{51}$, E.E. Kangal ${ }^{52}$, O. Kara, A. Kayis Topaksu, U. Kiminsu, M. Oglakci, G. Onengut ${ }^{53}$, K. Ozdemir ${ }^{54}$, A. Polatoz, U.G. Tok, H. Topakli ${ }^{50}$, S. Turkcapar, I.S. Zorbakir, C. Zorbilmez

Middle East Technical University, Physics Department, Ankara, Turkey

B. Bilin, G. Karapinar ${ }^{55}$, K. Ocalan ${ }^{56}$, M. Yalvac, M. Zeyrek

Bogazici University, Istanbul, Turkey

E. Gülmez, M. Kaya ${ }^{57}$, O. Kaya ${ }^{58}$, S. Tekten, E.A. Yetkin ${ }^{59}$

Istanbul Technical University, Istanbul, Turkey

M.N. Agaras, S. Atay, A. Cakir, K. Cankocak, I. Köseoglu

Institute for Scintillation Materials of National Academy of Science of Ukraine, Kharkov, Ukraine

B. Grynyov

National Scientific Center, Kharkov Institute of Physics and Technology, Kharkov, Ukraine

L. Levchuk

University of Bristol, Bristol, United Kingdom

F. Ball, L. Beck, J.J. Brooke, D. Burns, E. Clement, D. Cussans, O. Davignon, H. Flacher,

J. Goldstein, G.P. Heath, H.F. Heath, L. Kreczko, D.M. Newbold ${ }^{60}$, S. Paramesvaran,

T. Sakuma, S. Seif El Nasr-storey, D. Smith, V.J. Smith

Rutherford Appleton Laboratory, Didcot, United Kingdom

K.W. Bell, A. Belyaev ${ }^{61}$, C. Brew, R.M. Brown, L. Calligaris, D. Cieri, D.J.A. Cockerill, J.A. Coughlan, K. Harder, S. Harper, J. Linacre, E. Olaiya, D. Petyt, C.H. ShepherdThemistocleous, A. Thea, I.R. Tomalin, T. Williams

Imperial College, London, United Kingdom

G. Auzinger, R. Bainbridge, J. Borg, S. Breeze, O. Buchmuller, A. Bundock, S. Casasso, M. Citron, D. Colling, L. Corpe, P. Dauncey, G. Davies, A. De Wit, M. Della Negra, R. Di 
Maria, A. Elwood, Y. Haddad, G. Hall, G. Iles, T. James, R. Lane, C. Laner, L. Lyons, A.-M. Magnan, S. Malik, L. Mastrolorenzo, T. Matsushita, J. Nash, A. Nikitenko ${ }^{7}$,

V. Palladino, M. Pesaresi, D.M. Raymond, A. Richards, A. Rose, E. Scott, C. Seez,

A. Shtipliyski, S. Summers, A. Tapper, K. Uchida, M. Vazquez Acosta ${ }^{62}$, T. Virdee ${ }^{15}$,

N. Wardle, D. Winterbottom, J. Wright, S.C. Zenz

Brunel University, Uxbridge, United Kingdom

J.E. Cole, P.R. Hobson, A. Khan, P. Kyberd, I.D. Reid, L. Teodorescu, S. Zahid

Baylor University, Waco, U.S.A.

A. Borzou, K. Call, J. Dittmann, K. Hatakeyama, H. Liu, N. Pastika, C. Smith

Catholic University of America, Washington DC, U.S.A.

R. Bartek, A. Dominguez

The University of Alabama, Tuscaloosa, U.S.A.

A. Buccilli, S.I. Cooper, C. Henderson, P. Rumerio, C. West

Boston University, Boston, U.S.A.

D. Arcaro, A. Avetisyan, T. Bose, D. Gastler, D. Rankin, C. Richardson, J. Rohlf, L. Sulak,

D. Zou

Brown University, Providence, U.S.A.

G. Benelli, D. Cutts, A. Garabedian, M. Hadley, J. Hakala, U. Heintz, J.M. Hogan, K.H.M. Kwok, E. Laird, G. Landsberg, J. Lee, Z. Mao, M. Narain, J. Pazzini, S. Piperov, S. Sagir, R. Syarif, D. Yu

University of California, Davis, Davis, U.S.A.

R. Band, C. Brainerd, D. Burns, M. Calderon De La Barca Sanchez, M. Chertok, J. Conway, R. Conway, P.T. Cox, R. Erbacher, C. Flores, G. Funk, W. Ko, R. Lander, C. Mclean, M. Mulhearn, D. Pellett, J. Pilot, S. Shalhout, M. Shi, J. Smith, D. Stolp, K. Tos, M. Tripathi, Z. Wang

University of California, Los Angeles, U.S.A.

M. Bachtis, C. Bravo, R. Cousins, A. Dasgupta, A. Florent, J. Hauser, M. Ignatenko, N. Mccoll, S. Regnard, D. Saltzberg, C. Schnaible, V. Valuev

University of California, Riverside, Riverside, U.S.A.

E. Bouvier, K. Burt, R. Clare, J. Ellison, J.W. Gary, S.M.A. Ghiasi Shirazi, G. Hanson, J. Heilman, G. Karapostoli, E. Kennedy, F. Lacroix, O.R. Long, M. Olmedo Negrete, M.I. Paneva, W. Si, L. Wang, H. Wei, S. Wimpenny, B. R. Yates

University of California, San Diego, La Jolla, U.S.A.

J.G. Branson, S. Cittolin, M. Derdzinski, R. Gerosa, D. Gilbert, B. Hashemi, A. Holzner, D. Klein, G. Kole, V. Krutelyov, J. Letts, I. Macneill, M. Masciovecchio, D. Olivito, S. Padhi, M. Pieri, M. Sani, V. Sharma, S. Simon, M. Tadel, A. Vartak, S. Wasserbaech ${ }^{63}$, J. Wood, F. Würthwein, A. Yagil, G. Zevi Della Porta 
University of California, Santa Barbara - Department of Physics, Santa Barbara, U.S.A.

N. Amin, R. Bhandari, J. Bradmiller-Feld, C. Campagnari, A. Dishaw, V. Dutta, M. Franco Sevilla, F. Golf, L. Gouskos, R. Heller, J. Incandela, A. Ovcharova, H. Qu, J. Richman, D. Stuart, I. Suarez, J. Yoo

California Institute of Technology, Pasadena, U.S.A.

D. Anderson, A. Bornheim, J.M. Lawhorn, H.B. Newman, T. Nguyen, C. Pena, M. Spiropulu, J.R. Vlimant, S. Xie, Z. Zhang, R.Y. Zhu

Carnegie Mellon University, Pittsburgh, U.S.A.

M.B. Andrews, T. Ferguson, T. Mudholkar, M. Paulini, J. Russ, M. Sun, H. Vogel, I. Vorobiev, M. Weinberg

University of Colorado Boulder, Boulder, U.S.A.

J.P. Cumalat, W.T. Ford, F. Jensen, A. Johnson, M. Krohn, S. Leontsinis, T. Mulholland, K. Stenson, S.R. Wagner

Cornell University, Ithaca, U.S.A.

J. Alexander, J. Chaves, J. Chu, S. Dittmer, K. Mcdermott, N. Mirman, J.R. Patterson,

D. Quach, A. Rinkevicius, A. Ryd, L. Skinnari, L. Soffi, S.M. Tan, Z. Tao, J. Thom, J. Tucker, P. Wittich, M. Zientek

\section{Fermi National Accelerator Laboratory, Batavia, U.S.A.}

S. Abdullin, M. Albrow, M. Alyari, G. Apollinari, A. Apresyan, A. Apyan, S. Banerjee, L.A.T. Bauerdick, A. Beretvas, J. Berryhill, P.C. Bhat, G. Bolla ${ }^{\dagger}$, K. Burkett, J.N. Butler, A. Canepa, G.B. Cerati, H.W.K. Cheung, F. Chlebana, M. Cremonesi, J. Duarte, V.D. Elvira, J. Freeman, Z. Gecse, E. Gottschalk, L. Gray, D. Green, S. Grünendahl, O. Gutsche, R.M. Harris, S. Hasegawa, J. Hirschauer, Z. Hu, B. Jayatilaka, S. Jindariani, M. Johnson, U. Joshi, B. Klima, B. Kreis, S. Lammel, D. Lincoln, R. Lipton, M. Liu, T. Liu, R. Lopes De Sá, J. Lykken, K. Maeshima, N. Magini, J.M. Marraffino, D. Mason, P. McBride, P. Merkel, S. Mrenna, S. Nahn, V. O’Dell, K. Pedro, O. Prokofyev, G. Rakness, L. Ristori, B. Schneider, E. Sexton-Kennedy, A. Soha, W.J. Spalding, L. Spiegel, S. Stoynev, J. Strait, N. Strobbe, L. Taylor, S. Tkaczyk, N.V. Tran, L. Uplegger, E.W. Vaandering, C. Vernieri, M. Verzocchi, R. Vidal, M. Wang, H.A. Weber, A. Whitbeck University of Florida, Gainesville, U.S.A.

D. Acosta, P. Avery, P. Bortignon, D. Bourilkov, A. Brinkerhoff, A. Carnes, M. Carver, D. Curry, R.D. Field, I.K. Furic, S.V. Gleyzer, B.M. Joshi, J. Konigsberg, A. Korytov, K. Kotov, P. Ma, K. Matchev, H. Mei, G. Mitselmakher, K. Shi, D. Sperka, N. Terentyev, L. Thomas, J. Wang, S. Wang, J. Yelton

Florida International University, Miami, U.S.A.

Y.R. Joshi, S. Linn, P. Markowitz, J.L. Rodriguez

Florida State University, Tallahassee, U.S.A.

A. Ackert, T. Adams, A. Askew, S. Hagopian, V. Hagopian, K.F. Johnson, T. Kolberg, G. Martinez, T. Perry, H. Prosper, A. Saha, A. Santra, V. Sharma, R. Yohay 
Florida Institute of Technology, Melbourne, U.S.A.

M.M. Baarmand, V. Bhopatkar, S. Colafranceschi, M. Hohlmann, D. Noonan, T. Roy, F. Yumiceva

University of Illinois at Chicago (UIC), Chicago, U.S.A.

M.R. Adams, L. Apanasevich, D. Berry, R.R. Betts, R. Cavanaugh, X. Chen, O. Evdokimov, C.E. Gerber, D.A. Hangal, D.J. Hofman, K. Jung, J. Kamin, I.D. Sandoval Gonzalez, M.B. Tonjes, H. Trauger, N. Varelas, H. Wang, Z. Wu, J. Zhang

The University of Iowa, Iowa City, U.S.A.

B. Bilki ${ }^{64}$, W. Clarida, K. Dilsiz ${ }^{65}$, S. Durgut, R.P. Gandrajula, M. Haytmyradov, V. Khristenko, J.-P. Merlo, H. Mermerkaya ${ }^{66}$, A. Mestvirishvili, A. Moeller, J. Nachtman, H. Ogul ${ }^{67}$, Y. Onel, F. Ozok ${ }^{68}$, A. Penzo, C. Snyder, E. Tiras, J. Wetzel, K. Yi

Johns Hopkins University, Baltimore, U.S.A.

B. Blumenfeld, A. Cocoros, N. Eminizer, D. Fehling, L. Feng, A.V. Gritsan, P. Maksimovic, C. Mantilla, J. Roskes, U. Sarica, M. Swartz, M. Xiao, C. You

The University of Kansas, Lawrence, U.S.A.

A. Al-bataineh, P. Baringer, A. Bean, S. Boren, J. Bowen, J. Castle, S. Khalil, A. Kropivnitskaya, D. Majumder, W. Mcbrayer, M. Murray, C. Royon, S. Sanders, E. Schmitz, J.D. Tapia Takaki, Q. Wang

Kansas State University, Manhattan, U.S.A.

A. Ivanov, K. Kaadze, Y. Maravin, A. Mohammadi, L.K. Saini, N. Skhirtladze, S. Toda

Lawrence Livermore National Laboratory, Livermore, U.S.A.

F. Rebassoo, D. Wright

University of Maryland, College Park, U.S.A.

C. Anelli, A. Baden, O. Baron, A. Belloni, S.C. Eno, Y. Feng, C. Ferraioli, N.J. Hadley,

S. Jabeen, G.Y. Jeng, R.G. Kellogg, J. Kunkle, A.C. Mignerey, F. Ricci-Tam, Y.H. Shin,

A. Skuja, S.C. Tonwar

Massachusetts Institute of Technology, Cambridge, U.S.A.

D. Abercrombie, B. Allen, V. Azzolini, R. Barbieri, A. Baty, R. Bi, S. Brandt, W. Busza, I.A. Cali, M. D'Alfonso, Z. Demiragli, G. Gomez Ceballos, M. Goncharov, D. Hsu, M. Hu, Y. Iiyama, G.M. Innocenti, M. Klute, D. Kovalskyi, Y.S. Lai, Y.-J. Lee, A. Levin, P.D. Luckey, B. Maier, A.C. Marini, C. Mcginn, C. Mironov, S. Narayanan, X. Niu, C. Paus, C. Roland, G. Roland, J. Salfeld-Nebgen, G.S.F. Stephans, K. Tatar, D. Velicanu, J. Wang, T.W. Wang, B. Wyslouch

University of Minnesota, Minneapolis, U.S.A.

A.C. Benvenuti, R.M. Chatterjee, A. Evans, P. Hansen, J. Hiltbrand, S. Kalafut, Y. Kubota, Z. Lesko, J. Mans, S. Nourbakhsh, N. Ruckstuhl, R. Rusack, J. Turkewitz, M.A. Wadud

University of Mississippi, Oxford, U.S.A.

J.G. Acosta, S. Oliveros 
University of Nebraska-Lincoln, Lincoln, U.S.A.

E. Avdeeva, K. Bloom, D.R. Claes, C. Fangmeier, R. Gonzalez Suarez, R. Kamalieddin, I. Kravchenko, J. Monroy, J.E. Siado, G.R. Snow, B. Stieger

State University of New York at Buffalo, Buffalo, U.S.A.

J. Dolen, A. Godshalk, C. Harrington, I. Iashvili, D. Nguyen, A. Parker, S. Rappoccio, B. Roozbahani

Northeastern University, Boston, U.S.A.

G. Alverson, E. Barberis, C. Freer, A. Hortiangtham, A. Massironi, D.M. Morse, T. Orimoto, R. Teixeira De Lima, D. Trocino, T. Wamorkar, B. Wang, A. Wisecarver, D. Wood

Northwestern University, Evanston, U.S.A.

S. Bhattacharya, O. Charaf, K.A. Hahn, N. Mucia, N. Odell, M.H. Schmitt, K. Sung, M. Trovato, M. Velasco

University of Notre Dame, Notre Dame, U.S.A.

R. Bucci, N. Dev, M. Hildreth, K. Hurtado Anampa, C. Jessop, D.J. Karmgard, N. Kellams, K. Lannon, W. Li, N. Loukas, N. Marinelli, F. Meng, C. Mueller, Y. Musienko ${ }^{36}$, M. Planer, A. Reinsvold, R. Ruchti, P. Siddireddy, G. Smith, S. Taroni, M. Wayne, A. Wightman, M. Wolf, A. Woodard

The Ohio State University, Columbus, U.S.A.

J. Alimena, L. Antonelli, B. Bylsma, L.S. Durkin, S. Flowers, B. Francis, A. Hart, C. Hill, W. Ji, B. Liu, W. Luo, B.L. Winer, H.W. Wulsin

Princeton University, Princeton, U.S.A.

S. Cooperstein, O. Driga, P. Elmer, J. Hardenbrook, P. Hebda, S. Higginbotham, A. Kalogeropoulos, D. Lange, J. Luo, D. Marlow, K. Mei, I. Ojalvo, J. Olsen, C. Palmer, P. Piroué, D. Stickland, C. Tully

University of Puerto Rico, Mayaguez, U.S.A.

S. Malik, S. Norberg

Purdue University, West Lafayette, U.S.A.

A. Barker, V.E. Barnes, S. Das, S. Folgueras, L. Gutay, M.K. Jha, M. Jones, A.W. Jung, A. Khatiwada, D.H. Miller, N. Neumeister, C.C. Peng, H. Qiu, J.F. Schulte, J. Sun, F. Wang, R. Xiao, W. Xie

Purdue University Northwest, Hammond, U.S.A.

T. Cheng, N. Parashar, J. Stupak

Rice University, Houston, U.S.A.

Z. Chen, K.M. Ecklund, S. Freed, F.J.M. Geurts, M. Guilbaud, M. Kilpatrick, W. Li, B. Michlin, B.P. Padley, J. Roberts, J. Rorie, W. Shi, Z. Tu, J. Zabel, A. Zhang

University of Rochester, Rochester, U.S.A.

A. Bodek, P. de Barbaro, R. Demina, Y.t. Duh, T. Ferbel, M. Galanti, A. Garcia-Bellido, J. Han, O. Hindrichs, A. Khukhunaishvili, K.H. Lo, P. Tan, M. Verzetti 
The Rockefeller University, New York, U.S.A.

R. Ciesielski, K. Goulianos, C. Mesropian

Rutgers, The State University of New Jersey, Piscataway, U.S.A.

A. Agapitos, J.P. Chou, Y. Gershtein, T.A. Gómez Espinosa, E. Halkiadakis, M. Heindl,

E. Hughes, S. Kaplan, R. Kunnawalkam Elayavalli, S. Kyriacou, A. Lath, R. Montalvo,

K. Nash, M. Osherson, H. Saka, S. Salur, S. Schnetzer, D. Sheffield, S. Somalwar, R. Stone,

S. Thomas, P. Thomassen, M. Walker

University of Tennessee, Knoxville, U.S.A.

A.G. Delannoy, M. Foerster, J. Heideman, G. Riley, K. Rose, S. Spanier, K. Thapa

Texas A\&M University, College Station, U.S.A.

O. Bouhali ${ }^{69}$, A. Castaneda Hernandez ${ }^{69}$, A. Celik, M. Dalchenko, M. De Mattia, A. Delgado, S. Dildick, R. Eusebi, J. Gilmore, T. Huang, T. Kamon ${ }^{70}$, R. Mueller, Y. Pakhotin, R. Patel, A. Perloff, L. Perniè, D. Rathjens, A. Safonov, A. Tatarinov, K.A. Ulmer

\section{Texas Tech University, Lubbock, U.S.A.}

N. Akchurin, J. Damgov, F. De Guio, P.R. Dudero, J. Faulkner, E. Gurpinar, S. Kunori, K. Lamichhane, S.W. Lee, T. Libeiro, T. Mengke, S. Muthumuni, T. Peltola, S. Undleeb, I. Volobouev, Z. Wang

Vanderbilt University, Nashville, U.S.A.

S. Greene, A. Gurrola, R. Janjam, W. Johns, C. Maguire, A. Melo, H. Ni, K. Padeken, P. Sheldon, S. Tuo, J. Velkovska, Q. Xu

University of Virginia, Charlottesville, U.S.A.

M.W. Arenton, P. Barria, B. Cox, R. Hirosky, M. Joyce, A. Ledovskoy, H. Li, C. Neu, T. Sinthuprasith, Y. Wang, E. Wolfe, F. Xia

\section{Wayne State University, Detroit, U.S.A.}

R. Harr, P.E. Karchin, N. Poudyal, J. Sturdy, P. Thapa, S. Zaleski

University of Wisconsin - Madison, Madison, WI, U.S.A.

M. Brodski, J. Buchanan, C. Caillol, S. Dasu, L. Dodd, S. Duric, B. Gomber, M. Grothe, M. Herndon, A. Hervé, U. Hussain, P. Klabbers, A. Lanaro, A. Levine, K. Long, R. Loveless, T. Ruggles, A. Savin, N. Smith, W.H. Smith, D. Taylor, N. Woods

$\dagger$ : Deceased

1: Also at Vienna University of Technology, Vienna, Austria

2: Also at State Key Laboratory of Nuclear Physics and Technology, Peking University, Beijing, China

3: Also at IRFU, CEA, Université Paris-Saclay, Gif-sur-Yvette, France

4: Also at Universidade Estadual de Campinas, Campinas, Brazil

5: Also at Universidade Federal de Pelotas, Pelotas, Brazil

6: Also at Université Libre de Bruxelles, Bruxelles, Belgium

7: Also at Institute for Theoretical and Experimental Physics, Moscow, Russia

8: Also at Joint Institute for Nuclear Research, Dubna, Russia 
9: Now at Ain Shams University, Cairo, Egypt

10: Now at British University in Egypt, Cairo, Egypt

11: Now at Cairo University, Cairo, Egypt

12: Also at Université de Haute Alsace, Mulhouse, France

13: Also at Skobeltsyn Institute of Nuclear Physics, Lomonosov Moscow State University, Moscow, Russia

14: Also at Tbilisi State University, Tbilisi, Georgia

15: Also at CERN, European Organization for Nuclear Research, Geneva, Switzerland

16: Also at RWTH Aachen University, III. Physikalisches Institut A, Aachen, Germany

17: Also at University of Hamburg, Hamburg, Germany

18: Also at Brandenburg University of Technology, Cottbus, Germany

19: Also at MTA-ELTE Lendület CMS Particle and Nuclear Physics Group, Eötvös Loránd University, Budapest, Hungary

20: Also at Institute of Nuclear Research ATOMKI, Debrecen, Hungary

21: Also at Institute of Physics, University of Debrecen, Debrecen, Hungary

22: Also at Indian Institute of Technology Bhubaneswar, Bhubaneswar, India

23: Also at Institute of Physics, Bhubaneswar, India

24: Also at University of Visva-Bharati, Santiniketan, India

25: Also at University of Ruhuna, Matara, Sri Lanka

26: Also at Isfahan University of Technology, Isfahan, Iran

27: Also at Yazd University, Yazd, Iran

28: Also at Plasma Physics Research Center, Science and Research Branch, Islamic Azad University, Tehran, Iran

29: Also at Università degli Studi di Siena, Siena, Italy

30: Also at INFN Sezione di Milano-Bicocca; Università di Milano-Bicocca, Milano, Italy

31: Also at Purdue University, West Lafayette, U.S.A.

32: Also at International Islamic University of Malaysia, Kuala Lumpur, Malaysia

33: Also at Malaysian Nuclear Agency, MOSTI, Kajang, Malaysia

34: Also at Consejo Nacional de Ciencia y Tecnología, Mexico city, Mexico

35: Also at Warsaw University of Technology, Institute of Electronic Systems, Warsaw, Poland

36: Also at Institute for Nuclear Research, Moscow, Russia

37: Now at National Research Nuclear University 'Moscow Engineering Physics Institute' (MEPhI), Moscow, Russia

38: Also at St. Petersburg State Polytechnical University, St. Petersburg, Russia

39: Also at University of Florida, Gainesville, U.S.A.

40: Also at P.N. Lebedev Physical Institute, Moscow, Russia

41: Also at California Institute of Technology, Pasadena, U.S.A.

42: Also at Budker Institute of Nuclear Physics, Novosibirsk, Russia

43: Also at Faculty of Physics, University of Belgrade, Belgrade, Serbia

44: Also at University of Belgrade, Faculty of Physics and Vinca Institute of Nuclear Sciences, Belgrade, Serbia

45: Also at Scuola Normale e Sezione dell'INFN, Pisa, Italy

46: Also at National and Kapodistrian University of Athens, Athens, Greece

47: Also at Riga Technical University, Riga, Latvia

48: Also at Universität Zürich, Zurich, Switzerland

49: Also at Stefan Meyer Institute for Subatomic Physics (SMI), Vienna, Austria

50: Also at Gaziosmanpasa University, Tokat, Turkey

51: Also at Istanbul Aydin University, Istanbul, Turkey 
52: Also at Mersin University, Mersin, Turkey

53: Also at Cag University, Mersin, Turkey

54: Also at Piri Reis University, Istanbul, Turkey

55: Also at Izmir Institute of Technology, Izmir, Turkey

56: Also at Necmettin Erbakan University, Konya, Turkey

57: Also at Marmara University, Istanbul, Turkey

58: Also at Kafkas University, Kars, Turkey

59: Also at Istanbul Bilgi University, Istanbul, Turkey

60: Also at Rutherford Appleton Laboratory, Didcot, United Kingdom

61: Also at School of Physics and Astronomy, University of Southampton, Southampton, United Kingdom

62: Also at Instituto de Astrofísica de Canarias, La Laguna, Spain

63: Also at Utah Valley University, Orem, U.S.A.

64: Also at Beykent University, Istanbul, Turkey

65: Also at Bingol University, Bingol, Turkey

66: Also at Erzincan University, Erzincan, Turkey

67: Also at Sinop University, Sinop, Turkey

68: Also at Mimar Sinan University, Istanbul, Istanbul, Turkey

69: Also at Texas A\&M University at Qatar, Doha, Qatar

70: Also at Kyungpook National University, Daegu, Korea 